\title{
Viscosity Solutions of Systems of PDEs with Interconnected Obstacles and Multi-Modes Switching Problem
}

\author{
S.Hamadène* and M.A. Morlais ${ }^{\dagger}$
}

\begin{abstract}
This paper deals with existence and uniqueness, in viscosity sense, of a solution for a system of $m$ variational partial differential inequalities with inter-connected obstacles. A particular case of this system is the deterministic version of the Verification Theorem of the Markovian optimal $m$-states switching problem. The switching cost functions are arbitrary. This problem is connected with the valuation of a power plant in the energy market. The main tool is the notion of systems of reflected backward stochastic differential equations with oblique reflection.
\end{abstract}

AMS Classification subjects: 60G40 ; 62P20 ; 91B99;91B28 ; 35B37 ; 49L25.

Keywords: Real options; Backward stochastic differential equations; Snell envelope; Stopping times ; Switching; Viscosity solution of PDEs; Variational inequalities.

\section{Introduction}

The multi-modes switching problem is by now well documented both in the economics or mathematical literatures (see e.g. [4, 5, 6, 9, 10, 15, 18, 19, 20, 27, 28, 29], etc. and the references therein). The pioneering work of Brennan and Schwarz [4] deals with a two-modes switching problem describing the life cycle of an investment in the natural resource industry. A major switching problem of interest is related to the energy market. Actually let us consider a power plant which has several modes of production and which is put in a specific mode according to its profitability which depends on the electricity price in the market. The manager of the plant aims at maximizing its global profit. For this objective, she implements an optimal strategy $\delta^{*}$ which is a pair of two sequences $\left(\tau_{k}\right)_{k \geq 1}$ and $\left(\xi_{k}\right)_{k \geq 1}$ describing respectively the optimal successive switching times and modes. However switching the plant from one mode to another is not free and generates expenditures and, on the other hand, when the plant is in a specific mode it provides a profit which depends on that mode.

\footnotetext{
${ }^{*}$ Université du Maine, LMM, Avenue Olivier Messiaen, 72085 Le Mans, Cedex 9, France ; hamadene@univ-lemans.fr

${ }^{\dagger}$ Same address ; e-mail: Marie_Amelie.Morlais@univ-lemans.fr
} 
It is well-known that the optimal switching problem is related to systems of reflected backward stochastic differential equations (BSDEs for short) with inter-connected obstacles or oblique reflection (see e.g. [5, 10, 18, 19, 20, 27]) of the following type: $\forall i \in \mathcal{J}:=\{1, \ldots, m\}$,

$$
\left\{\begin{array}{l}
Y_{s}^{i}=h_{T}^{i}(\omega)+\int_{s}^{T} f_{i}(\omega, r) d r+K_{T}^{i}-K_{s}^{i}-\int_{s}^{T} Z_{r}^{i} d B_{r}, \forall s \leq T \\
Y_{s}^{i} \geq \max _{j \in \mathcal{J}^{-i}}\left\{Y_{s}^{j}-g_{i j}(\omega, s)\right\}, \forall s \leq T \\
\int_{0}^{T}\left(Y_{s}^{i}-\max _{j \in \mathcal{J}^{-i}}\left\{Y_{s}^{j}-g_{i j}(\omega, s)\right) d K_{s}^{i}=0,\right.
\end{array}\right.
$$

where $\mathcal{J}^{-i}=\mathcal{J}-\{i\}$. Actually it is shown in the aforementioned papers that $Y_{0}^{i}$ is the optimal profit if the plant is in mode $i$ at $t=0$, i.e., $\forall i \in \mathcal{J}$,

$$
Y_{0}^{i}=\sup _{\delta=\left(\tau_{k}, \xi_{k}\right)_{k \geq 0} \in \mathcal{A}_{0}^{i}} \mathbb{E}\left[h^{\delta}(T, \omega)+\int_{t}^{T} f_{\delta}(s, \omega) d s-\sum_{k \geq 0} g_{\xi_{k}, \xi_{k+1}}\left(\tau_{k+1}\right) \mathbf{1}_{\left[\tau_{k+1}<T\right]}\right]
$$

where, $\mathcal{A}_{0}^{i}$ stands for the set of all admissible strategies starting from mode $i$ at time $t=0, f_{\delta}$ the instantaneous profit per unit of time when $\delta$ is implemented, $g_{\xi_{k}, \xi_{k+1}}$ is the switching cost from mode $\xi_{k}$ to mode $\xi_{k+1}\left(\xi_{0}=i, \tau_{0}=0\right)$ and finally $h^{\delta}(T)$ is the terminal profit under $\delta$.

Additionally, if we are given the solution $\left(Y^{i}\right)_{i=1, \cdots, m}$ then the optimal strategy $\delta^{*}=\left(\xi_{k}^{*}, \tau_{k}^{*}\right)$ is uniquely characterized as follows: if we set $\tau_{1}^{*}=0$, and $\xi_{1}^{*}=i$ then for any $k \geq 1$

$$
\left\{\begin{array}{l}
\tau_{k+1}^{*}=\inf \left\{t>\tau_{k}^{*}, Y_{t}^{\xi_{k}^{*}}=\max _{j \in \mathcal{J}^{-\xi_{k}^{*}}}\left(Y_{t}^{j}-g_{\xi_{k}^{*}, j}(\omega, t)\right)\right\} \wedge T, \\
\xi_{k+1}^{*} \in \operatorname{argmax}\left\{j, Y_{t}^{\xi_{k}^{*}}=\max _{j \in \mathcal{J}^{-\xi_{k}^{*}}}\left(Y_{t}^{j}-g_{\xi_{k}^{*}, j}(\omega, t)\right)\right\} .
\end{array}\right.
$$

In the case of Markovian setting and when randomness stems from an exogenous Itô diffusion process $\left(X_{s}^{t, x}\right)_{s \leq T},(t, x) \in[0, T] \times \mathbb{R}^{k}$, of the following type:

$$
d X_{s}^{t, x}=b\left(s, X_{s}^{t, x}\right) d s+\sigma\left(s, X_{s}^{t, x}\right) d B_{s}, s \in[t, T] ; X_{s}^{t, x}=x \text { for } s \leq t,
$$

that is to say, the processes $f_{i}(s, \omega), h_{i}(s, \omega)$ and $g_{i j}(s, \omega)$ are deterministic functions of $\left(s, X_{s}^{t, x}(\omega)\right)$, the optimal switching problem is also related to the following system of variational inequalities with inter-connected obstacles: $\forall i \in \mathcal{J}$

$$
\left\{\begin{array}{l}
\min \left\{v_{i}(t, x)-\max _{j \in \mathcal{J}^{-i}}\left(-g_{i j}(t, x)+v_{j}(t, x)\right),-\partial_{t} v_{i}(t, x)-\mathcal{L} v_{i}(t, x)-f_{i}(t, x)\right\}=0 \\
v_{i}(T, x)=h_{i}(x)
\end{array}\right.
$$

where $\mathcal{L}$ is the infinitesimal generator associated with $X^{t, x}$. The process $X^{t, x}$ can be the electricity price in the market or the dynamics of factors which determine that price. System (1.4) is the deterministic version of the verification theorem of the optimal switching problem.

Actually in [15], the authors have proved that if, mainly, the switching costs satisfy $g_{i j}(t, x) \geq \gamma_{0}>0$ then the system (1.4) has a unique continuous solution $\left(v^{1}, \ldots, v^{m}\right)$ in viscosity sense and the following relationship holds true:

$$
\forall i \in \mathcal{J},(t, x) \in[0, T] \times \mathbb{R}^{k}, \forall s \in[t, T], Y_{s}^{i}=v^{i}\left(s, X_{s}^{t, x}\right)
$$


where $\left(Y^{i}\right)_{i \in \mathcal{J}}$ are the processes solution of (1.1) with $f_{i}, g_{i j}$ and $h_{i}$ which are deterministic functions of $\left(s, X_{s}^{t, x}\right)$.

So the main objective of this paper is to deal with system (1.4) in its general setting, i.e., to study the existence and uniqueness of a solution in viscosity sense for the following: $\forall i \in \mathcal{J}$

$$
\left\{\begin{array}{l}
\min \left\{v_{i}(t, x)-\max _{j \in \mathcal{J}^{-i}}\left(-g_{i j}(t, x)+v_{j}(t, x)\right),\right. \\
\left.\quad-\partial_{t} v_{i}(t, x)-\mathcal{L} v_{i}(t, x)-f_{i}\left(t, x, v^{1}(t, x), \ldots, v^{m}(t, x), \sigma^{\top}(t, x) D_{x} v^{i}(t, x)\right)\right\}=0 ; \\
v_{i}(T, x)=h_{i}(x) .
\end{array}\right.
$$

As previously pointed out, system (1.5) is connected with the following system of reflected BSDEs with oblique reflection :

$$
\left\{\begin{array}{l}
Y_{s}^{i ; t, x}=h^{i}\left(X_{T}^{t, x}\right)+\int_{s}^{T} f_{i}\left(r, X_{r}^{t, x}, Y_{r}^{1 ; t, x}, \ldots, Y_{r}^{m ; t, x}, Z_{r}^{i ; t, x}\right) d r+K_{T}^{i ; t, x}-K_{s}^{i ; t, x}-\int_{s}^{T} Z_{r}^{i ; t, x} d B_{r}, s \leq T ; \\
Y_{s}^{i ; t, x} \geq \max _{j \in \mathcal{J}^{-i}}\left\{Y_{s}^{j ; t, x}-g_{i j}\left(s, X_{s}^{t, x}\right)\right\}, s \leq T ; \\
\int_{0}^{T}\left(Y_{s}^{i ; t, x}-\max _{j \in \mathcal{J}^{-i}}\left\{Y_{s}^{j ; t, x}-g_{i j}\left(s, X_{s}^{t, x}\right)\right) d K_{s}^{i ; t, x}=0 .\right.
\end{array}\right.
$$

This system has already been studied in [7, 19] in this general form.

There are at least two motivations for considering this problem. The first one is to extend, as much as possible, the Feynman-Kac's representation of the solution of (1.6) via the solution of (1.5) and the process $X^{t, x}$. This issue is very important if we are willing to consider the numerical study of (1.6) especially in connection with the pricing of gas options in the energy market (one can see e.g. [2] for more details). The second one is that solving this problem is a step toward the study of zero-sum switching games which are encountered especially in the carbon market by energy firms (see e.g. [2] for more details).

The novelty of this paper lies in the fact that we investigate both existence and uniqueness of a continuous viscosity solution of (1.5) under the following relaxed hypotheses:

(i) the switching costs are non-negative and satisfy the no free loop condition (see [H3]-(ii) below)

(ii) for any $i \in \mathcal{J}$, either :

(a) $\forall k \in \mathcal{J}^{-i}$, the mappings $y_{k} \rightarrow f_{i}\left(t, x, y^{1}, \ldots, y^{k-1}, y^{k}, y^{k+1}, \ldots, y^{m}, z\right)$ are non-decreasing ; or

(b) $\forall k \in \mathcal{J}^{-i}$, the mappings $y_{k} \rightarrow f_{i}\left(t, x, y^{1}, \ldots, y^{k-1}, y^{k}, y^{k+1}, \ldots, y^{m}, z\right)$ are non-increasing.

In both cases we show existence of a solution for (1.5) while we have been able to show uniqueness only in the case (ii)-(a).

The closest paper to ours is the one by Elie-Kharroubi [11] where the authors deal also with the representation of solution of (1.6) by the viscosity solution of (1.5). However their approach, based on the minimal solutions of constrained BSDEs with jumps introduced in [23], is not very satisfactory since it induces assumptions on the data of the problem which are either not natural or difficult to 
verify in practice. Finally note that there are also works related to viscosity solutions of the switching problem but their settings and/or approaches are not the same as ours [1, 3, 20, 28], etc.

This paper is organized as follows:

In Section 2 we collect the main assumptions on the data of the problem and we define the notion of a viscosity solution for the system (1.5). In Section 3, we once more introduce the switching problem and provide some results related to solutions of systems of reflected BSDEs with oblique reflection which are rather new since they are obtained under weaker conditions than the ones of the literature on the subject (see e.g. [7, 19], etc.). In particular, we mainly deal with the non free loop property [H3]-(ii) on the switching costs. Those results are basic to deal with the main purpose of this work. In Section 4, we provide a comparison result between sub-solutions and super-solutions of the system (1.5) in the case when for any $i \in \mathcal{J}$ the function $f_{i}$ depends on $\left(y^{i}\right)_{i \in \mathcal{J}}$ only through $y^{i}$. We then show, in this specific framework of functions $f_{i}$, that system (1.5) has a unique continuous solution $\left(v^{i}(t, x)\right)_{i \in \mathcal{J}}$ which is moreover of polynomial growth. As a by-product, we provide a probabilistic representation for the solution of (1.6) via the deterministic continuous functions $\left(v^{i}(t, x)\right)_{i \in \mathcal{J}}$ and the process $X^{t, x}$. In Section 5, we deal with the general framework under mainly conditions (i)-(ii) above. Using the results of Sections 3 and 4, we construct in each case an approximating scheme which is convergent and whose limit is a solution in viscosity sense for system (1.5). Finally under condition (ii)-(a), we show that system (1.5) satisfies the comparison property between sub- and super-solutions. Thus under conditions (ii)-(a) the solution of system (1.5) is unique.

\section{Assumptions and problem formulation}

Let $T$ (resp. $k$ ) be a fixed real (resp. integer) positive constant, let $\mathcal{J}:=\{1, \ldots, m\}$ and let us consider the following functions: for $i, j \in \mathcal{J}$,

$$
\begin{aligned}
& b:(t, x) \in[0, T] \times \mathbb{R}^{k} \mapsto b(t, x) \in \mathbb{R}^{k} ; \\
& \sigma:(t, x) \in[0, T] \times \mathbb{R}^{k} \mapsto \sigma(t, x) \in \mathbb{R}^{k \times d} ; \\
& f_{i}:\left(t, x, y^{1}, \ldots, y^{m}, z\right) \in[0, T] \times \mathbb{R}^{k+m+d} \mapsto f_{i}\left(t, x, y^{1}, \ldots, y^{m}, z\right) \in \mathbb{R} ; \\
& g_{i j}:(t, x) \in[0, T] \times \mathbb{R}^{k} \mapsto g_{i j}(t, x) \in \mathbb{R}(i \neq j) ; \\
& h_{i}: x \in \mathbb{R}^{k} \mapsto h_{i}(x) \in \mathbb{R} .
\end{aligned}
$$

Next let $\phi:(t, x) \in[0, T] \times \mathbb{R}^{k} \mapsto \phi(t, x) \in \mathbb{R}$ be a function. It is called of polynomial growth if there exist two non negative real constants $C$ and $\gamma$ such that:

$$
|\phi(t, x)| \leq C\left(1+|x|^{\gamma}\right), \forall(t, x) \in[0, T] \times \mathbb{R}^{k} .
$$

Throughout this paper, we denote by $\Pi^{g}$ the class of functions with polynomial growth and by $\mathcal{C}^{1,2}\left([0, T] \times \mathbb{R}^{k}\right)$ (or simply $\mathcal{C}^{1,2}$ ) the set of functions defined on $[0, T] \times \mathbb{R}^{k}$ with values in $\mathbb{R}$ which are $C^{1}$ in $t$ and $C^{2}$ in $x$. 
We now consider the following assumptions:

[H1]: The functions $b$ and $\sigma$ are jointly continuous and of linear growth in $(t, x)$, and Lipschitz continuous w.r.t. $x$, i.e., there exists a constant $C \geq 0$ such that for any $t \in[0, T]$ and $x, x^{\prime} \in \mathbb{R}^{k}$

$$
|b(t, x)|+|\sigma(t, x)| \leq C(1+|x|) \quad \text { and } \quad\left|\sigma(t, x)-\sigma\left(t, x^{\prime}\right)\right|+\left|b(t, x)-b\left(t, x^{\prime}\right)\right| \leq C\left|x-x^{\prime}\right| .
$$

Throughout this paper we assume that assumption [H1] holds.

[H2]: for $i \in \mathcal{J}, f_{i}$ satisfies:

(i) $(t, x) \mapsto f_{i}\left(t, x, y^{1}, \ldots, y^{m}, z\right)$ is continuous uniformly w.r.t. $(\vec{y}, z):=\left(y^{1}, \ldots, y^{m}, z\right)$;

(ii) $f_{i}$ is uniformly Lipschitz continuous with respect to $(\vec{y}, z):=\left(y^{1}, \ldots, y^{m}, z\right)$, i.e., for some $C \geq 0$,

$$
\left|f_{i}\left(t, x, y^{1}, \ldots, y^{m}, z\right)-f_{i}\left(t, x, \bar{y}^{1}, \ldots, \bar{y}^{m}, \bar{z}\right)\right| \leq C\left(\left|y^{1}-\bar{y}^{1}\right|+\cdots+\left|y^{m}-\bar{y}^{m}\right|+|z-\bar{z}|\right)
$$

(iii) the mapping $(t, x) \mapsto f_{i}(t, x, 0, \ldots, 0)$ is $\mathcal{B}\left([0, T] \times \mathbb{R}^{k}\right)$-measurable and of polynomial growth i.e. it belongs to $\Pi^{g}$;

(iv) Monotonicity: $\forall i \in \mathcal{J}$, for any $k \in \mathcal{J}^{-i}$, the mapping $y_{k} \in \mathbb{R} \mapsto f_{i}\left(t, x, y_{1}, \ldots, y_{k-1}, y_{k}, y_{k+1}, \ldots, y_{m}\right)$ is non-decreasing whenever the other components $\left(t, x, y_{1}, \ldots, y_{k-1}, y_{k+1}, \ldots, y_{m}\right)$ are fixed;

[H3]: (i) $g_{i j}$ is jointly continuous in $(t, x)$, non-negative, i.e., $g_{i j}(t, x) \geq 0, \forall(t, x) \in[0, T] \times \mathbb{R}^{k}$ and belongs to $\Pi^{g}$;

(ii) The non-free loop property: for any $(t, x) \in[0, T] \times \mathbb{R}^{k}$ and for any sequence of indices $i_{1}, \ldots, i_{k}$ such that $i_{1}=i_{k}$ and $\operatorname{card}\left\{i_{1}, \ldots, i_{k}\right\}=k-1$ we have:

$$
g_{i_{1} i_{2}}(t, x)+g_{i_{2} i_{3}}(t, x)+\cdots+g_{i_{k-1} i_{k}}(t, x)+g_{i_{k} i_{1}}(t, x)>0, \forall(t, x) \in[0, T] \times \mathbb{R}^{k} .
$$

As a convention we assume hereafter that $g_{i i}(t, x)=0$ for any $(t, x) \in[0, T] \times \mathbb{R}^{k}$ and $i \in \mathcal{J}$.

[H4]: $h_{i}$ is continuous, belongs to $\Pi^{g}$ and satisfies:

$$
\forall x \in \mathbb{R}, h_{i}(x) \geq \max _{j \in \mathcal{J}^{-i}}\left(h_{j}(x)-g_{i j}((T, x))\right.
$$

Next let us introduce the following infinitesimal generator

$$
\mathcal{L} \varphi(t, x)=\frac{1}{2} \operatorname{Tr}\left[\left(\sigma . \sigma^{\top}\right)(t, x) D_{x x}^{2} \varphi(t, x)\right]+b(t, x)^{\top} \cdot D_{x} \varphi(t, x)
$$

for a function $\varphi$ which belongs to $\mathcal{C}^{1,2}\left([0, T] \times \mathbb{R}^{k} ; \mathbb{R}\right)\left(\operatorname{Tr}\right.$ is the trace of a symmetric matrix and $(.)^{\top}$ stands for the transpose). It is associated with a stochastic process which we will describe precisely below. 
In this paper we are concerned with the existence and uniqueness in viscosity sense of the solution $\left(v^{1}, \ldots, v^{m}\right):(t, x) \in[0, T] \times \mathbb{R}^{k} \mapsto\left(v^{1}(t, x), \ldots, v^{m}(t, x)\right) \in \mathbb{R}^{m}$ of the following system of $m$ partial differential equations with inter-connected obstacles: $\forall i \in \mathcal{J}$

$$
\left\{\begin{array}{l}
\min \left\{v_{i}(t, x)-\max _{j \in \mathcal{J}^{-i}}\left(-g_{i j}(t, x)+v_{j}(t, x)\right)\right. \\
\left.\quad-\partial_{t} v_{i}(t, x)-\mathcal{L} v_{i}(t, x)-f_{i}\left(t, x, v^{1}(t, x), \ldots, v^{m}(t, x), \sigma^{\top}(t, x) . D_{x} v^{i}(t, x)\right)\right\}=0 \\
v_{i}(T, x)=h_{i}(x)
\end{array}\right.
$$

To proceed we will precise the notion of a viscosity solution of the system (2.3). It will be done in terms of sub- and super-jets. So for any locally bounded function $u:(t, x) \in[0, T] \times \mathbb{R}^{k} \mapsto u(t, x) \in \mathbb{R}$, we define its lower semicontinuous (lsc for short) envelope $u_{*}$, and upper semicontinuous (usc for short) envelope $u^{*}$ in the following way:

$$
u_{*}(t, x)=\underset{\left(t^{\prime}, x^{\prime}\right) \rightarrow(t, x), t^{\prime}<T}{\varliminf^{\prime}} u\left(t^{\prime}, x^{\prime}\right) \text { and } u^{*}(t, x)=\varlimsup_{\left(t^{\prime}, x^{\prime}\right) \rightarrow(t, x), t^{\prime}<T} u\left(t^{\prime}, x^{\prime}\right) .
$$

Definition 1 : Subjects and superjets

(i) For a function $u:[0, T] \times \mathbb{R}^{k} \rightarrow \mathbb{R}$, lsc (resp. usc), we denote by $J^{-} u(t, x)$ the parabolic subjet (resp. $J^{+} u(t, x)$ the parabolic superjet) of $u$ at $(t, x) \in[0, T] \times \mathbb{R}^{k}$, as the set of triples $(p, q, M) \in \mathbb{R} \times \mathbb{R}^{k} \times \mathbb{S}^{k}$ satisfying

$$
\begin{array}{r}
u\left(t^{\prime}, x^{\prime}\right) \geq(\operatorname{resp.} \leq) u(t, x)+p\left(t^{\prime}-t\right)+<q, x^{\prime}-x>+ \\
\quad \frac{1}{2}<x^{\prime}-x, M\left(x^{\prime}-x\right)>+o\left(\left|t^{\prime}-t\right|+\left|x^{\prime}-x\right|^{2}\right)
\end{array}
$$

where $\mathbb{S}^{k}$ is the set of symmetric real matrices of dimension $k$.

(ii) For a function $u:[0, T] \times \mathbb{R}^{k} \rightarrow \mathbb{R}$, lsc (resp. usc), we denote by $\bar{J}^{-} u(t, x)$ the parabolic limiting subjet (resp. $\bar{J}^{+} u(t, x)$ the parabolic limiting superjet) of $u$ at $(t, x) \in[0, T] \times \mathbb{R}^{k}$, as the set of triples $(p, q, M) \in \mathbb{R} \times \mathbb{R}^{k} \times \mathbb{S}^{k}$ such that:

$$
\begin{array}{r}
(p, q, M)=\lim _{n}\left(p_{n}, q_{n}, M_{n}\right), \quad(t, x)=\lim _{n}\left(t_{n}, x_{n}\right) \\
\text { with }\left(p_{n}, q_{n}, M_{n}\right) \in J^{-} u\left(t_{n}, x_{n}\right)\left(\text { resp. } J^{+} u\left(t_{n}, x_{n}\right)\right) \quad \text { and } u(t, x)=\lim _{n} u\left(t_{n}, x_{n}\right) .
\end{array}
$$

We now give the definition of a viscosity solution for the system of PDE equations with oblique reflection (2.3).

Definition 2 : Viscosity solution to (2.3)

(i) A function $\left(v_{1}, \ldots, v_{m}\right):[0, T] \times \mathbb{R}^{k} \rightarrow \mathbb{R}^{m}$ such that for any $i \in \mathcal{J}$, $v_{i}$ is lsc (resp. usc), is called a viscosity supersolution (resp. subsolution) to (2.3) if for any $i \in \mathcal{J}$, for any $(t, x) \in[0, T) \times \mathbb{R}^{k}$ and 
any $(p, q, M) \in \bar{J}^{-} v_{i}(t, x)\left(\right.$ resp. $\left.\bar{J}^{+} v^{i}(t, x)\right)$ we have:

$$
\left\{\begin{array}{l}
\min \left\{v_{i}(t, x)-\max _{j \in \mathcal{J}^{-i}}\left(-g_{i j}(t, x)+v_{j}(t, x)\right) ;\right. \\
\left.\quad-p-b(t, x)^{\top} \cdot q-\frac{1}{2} \operatorname{Tr}\left[\left(\sigma \sigma^{\top}\right)(t, x) M\right]-f_{i}\left(t, x, v_{1}, \ldots, v_{m}, \sigma(t, x)^{\top} \cdot M\right)\right\} \geq 0(\text { resp. } \leq 0) ; \\
v_{i}(T, x) \geq(\text { resp. } \leq) h_{i}(x) .
\end{array}\right.
$$

(ii) A locally bounded function $\left(v_{1}, \ldots, v_{m}\right):[0, T] \times \mathbb{R}^{k} \rightarrow \mathbb{R}^{m}$ is called a viscosity solution to (2.3) if $\left(v_{1 *}, \ldots, v_{m *}\right)$ (resp. $\left.\left(v_{1}^{*}, \ldots, v_{m}^{*}\right)\right)$ is a viscosity supersolution (resp. subsolution) of (2.3).

As pointed out previously we will show that system (2.3) has a unique solution in viscosity sense. A particular case of this system is the deterministic version of the optimal $m$-states switching problem which is well documented e.g. in [10, 20] and which we will describe in the next section.

\section{The optimal $m$-states switching problem}

\subsection{Setting of the problem}

Let $(\Omega, \mathcal{F}, \mathbb{P})$ be a fixed probability space on which is defined a standard $d$-dimensional Brownian motion $B=\left(B_{t}\right)_{0 \leq t \leq T}$ whose natural filtration is $\left(\mathcal{F}_{t}^{0}:=\sigma\left\{B_{s}, s \leq t\right\}\right)_{0 \leq t \leq T}$. Let $\mathbf{F}=\left(\mathcal{F}_{t}\right)_{0 \leq t \leq T}$ be the completed filtration of $\left(\mathcal{F}_{t}^{0}\right)_{0 \leq t \leq T}$ with the $\mathbb{P}$-null sets of $\mathcal{F}$, hence $\left(\mathcal{F}_{t}\right)_{0 \leq t \leq T}$ satisfies the usual conditions, i.e., it is right continuous and complete. Furthermore, let:

- $\mathcal{P}$ be the $\sigma$-algebra on $[0, T] \times \Omega$ of $\mathbf{F}$-progressively measurable sets ;

- $\mathcal{H}^{2, k}$ be the set of $\mathcal{P}$-measurable, $\mathbb{R}^{k}$-valued processes $w=\left(w_{t}\right)_{t \leq T}$ such that $\mathbb{E}\left[\int_{0}^{T}\left|w_{s}\right|^{2} d s\right]<\infty$;

- $\mathcal{S}^{2}$ be the set of $\mathcal{P}$-measurable, continuous, $\mathbb{R}$-valued processes $w=\left(w_{t}\right)_{t \leq T}$ such that $\mathbb{E}\left[\sup _{t \leq T}\left|w_{t}\right|^{2}\right]<\infty$.

The problem of multiple switching can be described through an example as follows. Assume we have a plant which produces a commodity, e.g. a power station which produces electricity. Let $\mathcal{J}$ be the set of all possible activity modes of the production of the commodity. A management strategy of the plant consists, on the one hand, of the choice of a sequence of nondecreasing stopping times $\left(\tau_{n}\right)_{n \geq 1}$ (i.e. $\tau_{n} \leq \tau_{n+1}$ and $\tau_{0}=0$ ) where the manager decides to switch the activity from its current mode to another one. On the other hand, it consists of the choice of the mode $\xi_{n}$, a r.v. $\mathcal{F}_{\tau_{n}}$-measurable with values in $\mathcal{J}$, to which the production is switched at $\tau_{n}$ from its current mode. Therefore the admissible management strategies of the plant are the pairs $(\delta, \xi):=\left(\left(\tau_{n}\right)_{n \geq 1},\left(\xi_{n}\right)_{n \geq 1}\right)$ for which we require also that $\mathbb{P}\left[\tau_{n}<T, \forall n \geq 0\right]=0$. This set is called of admissible strategies and denoted by $\mathcal{D}$.

Next, assuming that the production activity is in mode 1 at the initial time $t=0$, let $\left(\alpha_{t}\right)_{t \leq T}$ denote the indicator of the production activity's mode at time $t \in[0, T]$, i.e.,

$$
\alpha_{t}=\mathbb{1}_{\left[0, \tau_{1}\right]}(t)+\sum_{n \geq 1} \xi_{n} \mathbb{1}_{\left(\tau_{n}, \tau_{n+1}\right]}(t) .
$$


Finally, for $i \in \mathcal{J}$, let $\left(\psi_{i}(t, \omega)\right)_{t \leq T}$ be a process of $\mathcal{H}^{2,1}$ which stands for the instantaneous profit when the system is in state $i$ and for $i, j \in \mathcal{J}, i \neq j$, let $\left(g_{i j}(t, \omega)\right)_{t \leq T}$ be a process of $\mathcal{S}^{2}$ which denotes the switching cost of the production at time $t$ from current mode $i$ to another one $j$. If the plant is run under the admissible strategy $(\delta, \xi)=\left(\left(\tau_{n}\right)_{n \geq 1},\left(\xi_{n}\right)_{n \geq 1}\right)$ the expected total profit is given by:

$$
J(\delta, \xi):=\mathbb{E}\left[\int_{0}^{T} \psi_{\alpha_{s}}(s) d s-\sum_{n \geq 1} g_{\xi_{n-1}, \xi_{n}}\left(\tau_{n}\right) \mathbb{1}_{\left[\tau_{n}<T\right]}\right] .
$$

Therefore in several works authors are usually interested in either finding an optimal strategy, i.e, a strategy $\left(\delta^{*}, \xi^{*}\right)$ such that $J\left(\delta^{*}, \xi^{*}\right) \geq J(\delta, \xi)$ for any $(\delta, \xi) \in \mathcal{D}([10,20])$ or at least in characterizing the quantity $\sup _{(\delta, \xi) \in \mathcal{D}} J(\delta, \xi)$ in some specific cases $([5,15])$. This latter quantity is in a way the price of the power plant in the energy market.

\subsection{Connection with systems of reflected BSDEs with oblique reflection}

In order to tackle the switching problem described above, we usually relate it to systems of reflected BSDEs with oblique reflection which we introduce below in the case we need in order to deal also with the system of PDEs (2.3).

Let $(t, x) \in[0, T] \times \mathbb{R}^{k}$ and $\left(X_{s}^{t, x}\right)_{s \leq T}$ be the solution of the following stochastic differential equation:

$$
d X_{s}^{t, x}=b\left(s, X_{s}^{t, x}\right) d s+\sigma\left(s, X_{s}^{t, x}\right) d B_{s}, s \in[t, T] \text { and } X_{s}^{t, x}=x \text { for } s \in[0, t] .
$$

The solution of this equation exists, is unique, since $b$ and $\sigma$ verify [H1], and satisfies:

$$
\forall p \geq 1, \mathbb{E}\left[\sup _{s \leq T}\left|X_{s}^{t, x}\right|^{p}\right] \leq C\left(1+|x|^{p}\right) .
$$

Next let us introduce the solution of the system of reflected BSDEs with oblique reflection associated with the deterministic functions $\left(\left(f_{i}\right)_{i \in \mathcal{J}},\left(g_{i j}\right)_{i, j \in \mathcal{J}},\left(h_{i}\right)_{i \in \mathcal{J}}\right)$ introduced in Section 2. The solution consists of $m$ triplets of processes $\left(\left(Y^{i ; t, x}, Z^{i ; t, x}, K^{i ; t, x}\right)\right)_{i \in \mathcal{J}}$, which is denoted, for convenience, by $\left(\left(Y^{i}, Z^{i}, K^{i}\right)\right)_{i \in \mathcal{J}}$ and satisfies: for any $i \in \mathcal{J}$,

$$
\left\{\begin{array}{l}
Y^{i}, K^{i} \in \mathcal{S}^{2}, Z^{i} \in \mathcal{H}^{2, d} \text { and } K^{i} \text { non-decreasing and } K_{0}^{i}=0 ; \\
Y_{s}^{i}=h_{i}\left(X_{T}^{t, x}\right)+\int_{s}^{T} f_{i}\left(r, X_{r}^{t, x}, Y_{r}^{1}, \ldots, Y_{r}^{m}, Z_{r}^{i}\right) d r+K_{T}^{i}-K_{s}^{i}-\int_{s}^{T} Z_{r}^{i} d B_{r}, \forall s \leq T \\
Y_{s}^{i} \geq \max _{j \in \mathcal{J}^{-i}}\left\{Y_{s}^{j}-g_{i j}\left(s, X_{s}^{t, x}\right)\right\}, \forall s \leq T \\
\int_{0}^{T}\left(Y_{s}^{i}-\max _{j \in \mathcal{J}^{-i}}\left\{Y_{s}^{j}-g_{i j}\left(s, X_{s}^{t, x}\right)\right\}\right) d K_{s}^{i}=0 .
\end{array}\right.
$$

We first provide an existence and uniqueness results of the solution of (3.3) and some of their properties as well.

\section{Theorem 1 Assume that:}

1) the functions $\left(f_{i}\right)_{i \in \mathcal{J}}$ satisfy (H2)-(ii), (iii) and (iv);

2) For any $i, j \in \mathcal{J}$, the functions $g_{i j}$ (resp. $h_{i}$ ) verify (H3) (resp. (H4)).

Then the system (3.3) has a solution $\left(\left(Y^{i}, Z^{i}, K^{i}\right)\right)_{i=1, m}$. 
Proof: Since the above assumptions are not exactly the same as the ones of Theorem 3.2 in [19] we give its main steps for sake of completeness.

Step 1: Let us consider the following BSDEs:

$$
\left\{\begin{array}{l}
\bar{Y} \in \mathcal{S}^{2}, \bar{Z} \in \mathcal{H}^{2, d} \\
\bar{Y}_{s}=\max _{i=1, m} h_{i}\left(X_{T}^{t, x}\right)+\int_{s}^{T}\left[\max _{i=1, m} f_{i}\right]\left(r, X_{r}^{t, x}, \bar{Y}_{r}, \ldots, \bar{Y}_{r}, \bar{Z}_{r}\right) d r-\int_{s}^{T} \bar{Z}_{r} d B_{r}, s \leq T,
\end{array}\right.
$$

and

$$
\left\{\begin{array}{l}
\underline{\mathrm{Y}} \in \mathcal{S}^{2}, \underline{\mathrm{Z}} \in \mathcal{H}^{2, d} \\
\underline{\mathrm{Y}}_{s}=\min _{i=1, m} h_{i}\left(X_{T}^{t, x}\right)+\int_{s}^{T}\left[\min _{i=1, m} f_{i}\right]\left(r, X_{r}^{t, x}, \underline{\mathrm{Y}}_{r}, \ldots, \underline{\mathrm{Y}}_{r}, \underline{\mathrm{Z}}_{r}\right) d r-\int_{s}^{T} \underline{\mathrm{Z}}_{r} d B_{r}, s \leq T .
\end{array}\right.
$$

Thanks to the result by Pardoux-Peng [24, the solutions of both (3.4) and (3.5) exist. We next introduce the following sequences of BSDEs defined recursively by: for any $i \in \mathcal{J}, Y^{i, 0}=\underline{Y}$ and for $n \geq 1$ and $s \leq T$,

$$
\left\{\begin{array}{l}
Y^{i, n}, K^{i, n} \in \mathcal{S}^{2}, Z^{i, n} \in \mathcal{H}^{2, d} \text { and } K^{i, n} \text { non-decreasing; } \\
Y_{s}^{i, n}=h_{i}\left(X_{T}^{t, x}\right)+\int_{s}^{T} f_{i}\left(r, X_{r}^{t, x}, Y_{r}^{1, n-1}, \ldots, Y_{r}^{i-1, n-1}, Y_{r}^{i, n}, Y_{r}^{i+1, n-1}, \ldots, Y_{r}^{m, n-1}, Z_{r}^{i, n}\right) d r \\
\quad+K_{T}^{i, n}-K_{s}^{i, n}-\int_{s}^{T} Z_{r}^{i, n} d B_{r} \\
Y_{s}^{i, n} \geq \max _{j \in \mathcal{J}^{-i}}\left\{Y_{s}^{j, n-1}-g_{i j}\left(s, X_{s}^{t, x}\right)\right\} \\
\int_{0}^{T}\left(Y_{s}^{i, n}-\max _{j \in \mathcal{J}^{-i}}\left\{Y_{s}^{j, n-1}-g_{i j}\left(s, X_{s}^{t, x}\right)\right\}\right) d K_{s}^{i, n}=0 .
\end{array}\right.
$$

By an induction argument and the result by El-Karoui et al. ([12], Theorem 5.2), we claim that the processes $\left(Y^{i, n}, Z^{i, n}, K^{i, n}\right)$ exist for any $n \geq 1$. Next using the comparison theorem of solutions of BSDEs (see e.g. Theorem 2.2 in [13]) we deduce that for any $i \in \mathcal{J}, Y^{i, 0} \leq Y^{i, 1}$. Now since $f_{i}$ satisfies the monotonicity property (H2)-(iv) and using once more the comparison of solutions of reflected BSDEs (see e.g. Theorem 4.1 in [12]) we obtain by induction that:

$$
\forall n \geq 0 \text { and } i \in \mathcal{J}, Y^{i, n} \leq Y^{i, n+1}
$$

Since the processes $((\bar{Y}, \bar{Z}, 0))_{i \in \mathcal{J}}$ is a solution for the system of obliquely reflected BSDEs associated with $\left(\left(\left[\max _{i=1, m} f_{i}\right]\left(s, X_{s}^{t, x}, y^{1}, \ldots, y^{m}, z\right)\right)_{i \in \mathcal{J}},\left(\max _{i=1, m} h_{i}\left(X_{T}^{t, x}\right)\right)_{i \in \mathcal{J}},\left(g_{i j}\left(s, X_{s}^{t, x}\right)\right)_{i, j \in \mathcal{J}}\right)$, an induction procedure and the repeated use of comparison theorem, which is justified taking into account that $f_{i}$ satisfies the monotonicity property (H2)-(iv)), leads to

$$
\forall n \geq 0, \quad \forall i \in \mathcal{J}, Y^{i, n} \leq \bar{Y}
$$

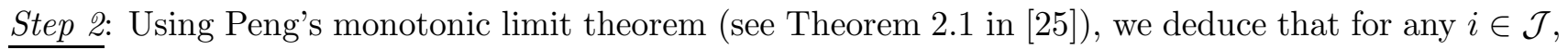
there exist:

(i) a càdlàg (for right continuous with left limits) process $Y^{i}$ such that $Y^{i, n} \nearrow Y^{i}$ pointwisely ; 
(ii) a process $Z^{i}$ of $\mathcal{H}^{2, d}$ such that, at least for a subsequence, $\left(Z^{i, n}\right)_{n \geq 0}$ converges weakly to $Z^{i}$ in $\mathcal{H}^{2, d}$ and strongly in $L^{p}(d t \otimes d P)$ for any $p \in[1,2[$;

(iii) a càdlàg non decreasing process $K^{i}$ such that for any stopping time $\tau,\left(K_{\tau}^{i, n}\right)_{n \geq 0}$ converges to $K_{\tau}^{i}$ in $L^{p}(d P)$ for any $p \in[1,2[$.

Additionally in taking the limit in (3.6), the triple of processes $\left(Y^{i}, Z^{i}, K^{i}\right)$ satisfies:

$$
\left\{\begin{array}{l}
Y_{s}^{i}=h_{i}\left(X_{T}^{t, x}\right)+\int_{s}^{T} f_{i}\left(r, X_{r}^{t, x}, Y_{r}^{1}, \ldots, Y_{r}^{m}, Z_{r}^{i}\right) d r+K_{T}^{i}-K_{s}^{i}-\int_{s}^{T} Z_{r}^{i} d B_{r} \text { and } \\
Y_{s}^{i} \geq \max _{j \in \mathcal{J}^{-i}}\left\{Y_{s}^{j}-g_{i j}\left(s, X_{s}^{t, x}\right)\right\}, \forall s \leq T
\end{array}\right.
$$

Next let us consider the following $m$ independent reflected BSDEs with a càdlàg barrier which, in addition, are independent of each other (for existence and uniqueness results for such BSDEs, we refer to [16]):

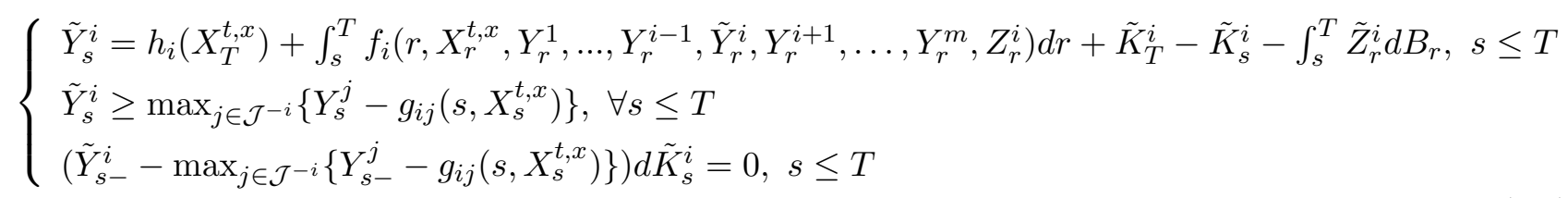

where $\tilde{Y}_{t-}^{i}\left(\operatorname{resp} . K_{t-}^{i}\right)=\lim _{s} \nearrow_{t} \tilde{Y}_{s}^{i}\left(\right.$ resp. $\left.K_{s}^{i}\right), t>0$. Henceforth in using once more comparison theorem (e.g. [16], Theorem 1.5) we have for any $i \in \mathcal{J}, Y^{i, n} \leq \tilde{Y}^{i}$ and then $Y^{i} \leq \tilde{Y}^{i}$. On the other hand using Itô's formula with $\left(\left(\tilde{Y}_{t}^{i}-Y_{t}^{i}\right)^{+}\right)^{2}, t \leq T$, we obtain that $\tilde{Y}_{t}^{i} \leq Y_{t}^{i}$, for any $t \leq T$. Actually this is true because $\tilde{Y}^{i}$ is the minimal solution which satisfies system (3.7). It implies that $\tilde{Y}^{i}=Y^{i}$, and then $Z^{i}=\tilde{Z}^{i}, \tilde{K}^{i}=K^{i}$, for any $i \in \mathcal{J}$. Therefore $\left(Y^{i}, Z^{i}, K^{i}\right)$ satisfies not only the system (3.7) but also:

$$
\int_{0}^{T}\left(Y_{s-}^{i}-\max _{j \in \mathcal{J}^{-i}}\left\{Y_{s-}^{j}-g_{i j}\left(s, X_{s}^{t, x}\right)\right\}\right) d K_{s}^{i}=0, \forall i \in \mathcal{J}
$$

It remains to show that, for each $i \in \mathcal{J}, Y^{i}$ is continuous. For this, let us assume that for some $i$ and $t_{0}$, $\Delta Y_{t_{0}}^{i}:=\left(Y_{t_{0}}^{i}-Y_{t_{0}-}^{i}\right)<0$, therefore $\Delta K_{t_{0}}^{i}>0$ and then from (3.8) we deduce the existence of $i_{1} \in \mathcal{J}^{-i}$ such that $Y_{t_{0}-}^{i}=Y_{t_{0}-}^{i_{1}}-g_{i i_{1}}\left(t_{0}, X_{t_{0}}^{t, x}\right)$ and as $Y_{t_{0}}^{i} \geq Y_{t_{0}}^{i_{1}}-g_{i i_{1}}\left(t_{0}, X_{t_{0}}^{t, x}\right)$ then $\Delta Y_{t_{0}}^{i_{1}}<0$. Repeating this reasoning as many times as necessary we deduce the existence of a sequence of indices $\left(i_{k}\right)$ such that $i_{k} \in \mathcal{J}^{-i_{k-1}}$ and

$$
Y_{t_{0}-}^{i_{k-1}}=Y_{t_{0}-}^{i_{k}}-g_{i_{k-1} i_{k}}\left(t_{0}, X_{t_{0}}^{t, x}\right) .
$$

As $\mathcal{J}$ is finite then there exists a sequence $j_{1}, . ., j_{p-1}$ which are all different such that

$$
g_{j_{1} j_{2}}\left(t_{0}, X_{t_{0}}^{t, x}\right)+\cdots+g_{j_{p-1} j_{1}}\left(t_{0}, X_{t_{0}}^{t, x}\right)=0 .
$$

But this is contradictory with the assumption [H3]-(ii) and then such a $t_{0}$ does not exist and $Y^{i}$ is continuous for any $i \in \mathcal{J}$. Therefore $\left(\left(Y^{i}, Z^{i}, K^{i}\right)\right)_{i \in \mathcal{J}}$, is a solution for the system of reflected BSDEs (3.3). 
We now give a remark related to comparison of the solutions of system (3.3) constructed in Theorem 1. Its proof is rather easy since an induction argument allows to compare the solutions of the convergence schemes. Actually we have:

Remark 1 : Let $\left(f_{i}^{\prime}\right)_{i \in \mathcal{J}}$ (resp. $\left(g_{i j}^{\prime}\right)_{i, j \in \mathcal{J}}$, resp. $\left.\left(h_{i}^{\prime}\right)_{i \in \mathcal{J}}\right)$ be functions that satisfy (H2)-(ii), (iii), (iv) (resp. (H3), resp. (H4)) and let $\left(\left(Y^{\prime i}, Z^{\prime i}, K^{\prime i}\right)\right)_{i \in \mathcal{J}}$ be the solution of the system of reflected BSDEs associated with $\left(\left(f_{i}^{\prime}\right)_{i \in \mathcal{J}},\left(g_{i j}^{\prime}\right)_{i, j \in \mathcal{J}},\left(h_{i}^{\prime}\right)_{i \in \mathcal{J}}\right)$ constructed as in Theorem [1. If for any $i, j \in \mathcal{J}$ we have:

$$
f_{i} \leq f_{i}^{\prime}, h_{i} \leq h_{i}^{\prime} \text { and } g_{i j} \geq g_{i j}^{\prime}
$$

then for any $i \in \mathcal{J}$,

$$
Y^{i} \leq Y^{\prime i}
$$

In case of uniqueness of the solutions of those systems, this result reduces to the comparison of the solutions.

We now focus on the regularity properties of the solution of system (3.3) constructed in Theorem 1.

Proposition 3.1 Assume the assumptions of Theorem 1 are fulfilled. Then there exist lsc deterministic functions $\left(v^{i}\right)_{i \in \mathcal{J}}$, defined on $[0, T] \times \mathbb{R}^{k}, \mathbb{R}$-valued and which belong to $\Pi^{g}$, such that:

$$
\forall i \in \mathcal{J}, \forall s \in[t, T], Y_{s}^{i}=v^{i}\left(s, X_{s}^{t, x}\right),
$$

where $\left(\left(Y^{i}, Z^{i}, K^{i}\right)\right)_{i \in \mathcal{J}}$ is the solution of (3.3) constructed in Theorem 1 .

Proof: Actually under the hypotheses of Theorem 1, there exist deterministic continuous with polynomial growth functions $\bar{v}(t, x)$ and $\underline{v}(t, x)$ with values in $\mathbb{R}$ such that for any $s \in[t, T], \bar{Y}_{s}=\bar{v}\left(s, X_{s}^{t, x}\right)$ and $\underline{Y}_{s}=\underline{v}\left(s, X_{s}^{t, x}\right)([13]$, Th.4.1).

Next by induction and thanks to the result by El-Karoui et al. ([12], pp.729), there exist deterministic continuous functions $v^{i, n}(t, x)$ in the class $\Pi^{g}$ such that for any $i \in \mathcal{J}$ and $n \geq 0$,

$$
Y_{s}^{i, n}=v^{i, n}\left(s, X_{s}^{t, x}\right), \forall s \in[t, T]
$$

the process $Y^{i, n}$ being defined as the unique solution of (3.6) (see step 1, Theorem 10) As $Y^{i, n} \leq Y^{i, n+1} \leq$ $\bar{Y}$ then for fixed $i$, the sequence $\left(v^{i, n}\right)_{n \geq 0}$ is non-decreasing and such that $v^{i, n} \leq \bar{v}$, then it converges pointwisely to $v^{i}$. This latter function is therefore lower semi-continuous on $[0, T] \times \mathbb{R}^{k}$, of polynomial growth since $\underline{v} \leq v^{i} \leq \bar{v}$ and finally for any $s \in[t, T], Y_{s}^{i}=v^{i}\left(s, X_{s}^{t, x}\right)$.

Remark 2 : Since $v^{i}, i \in \mathcal{J}$, belongs to $\Pi^{g}$, then classically (see e.g. [12]) one can show that for any $i \in \mathcal{J},\left\|Z^{i}\right\|_{\mathcal{H}^{2, d}}(t, x)$ is also of polynomial growth. 
We next give a representation result for the solutions of system (3.3) and, as a by product, we obtain a uniqueness result in some specific cases. Actually let fix $\vec{u}:=\left(u^{i}\right)_{i=1, m}$ in $\mathcal{H}^{2, m}$ and let us consider the following system of reflected BSDEs with oblique reflection:

$$
\forall i \in \mathcal{J}, \forall s \leq T
$$

$$
\left\{\begin{array}{l}
Y^{u, i}, K^{u, i} \in \mathcal{S}^{2}, Z^{u, i} \in \mathcal{H}^{2, d} \text { and } K^{u, i} \text { non-decreasing }\left(K_{0}^{u, i}=0\right) ; \\
Y_{s}^{u, i}=h_{i}\left(X_{T}^{t, x}\right)+\int_{s}^{T} f_{i}\left(r, X_{r}^{t, x}, \overrightarrow{u_{r}}, Z_{r}^{u, i}\right) d r+K_{T}^{u, i}-K_{s}^{u, i}-\int_{s}^{T} Z_{r}^{u, i} d B_{r} \\
Y_{s}^{u, i} \geq \max _{j \in \mathcal{J}^{-i}}\left\{Y_{s}^{u, j}-g_{i j}\left(s, X_{s}^{t, x}\right)\right\} \\
\int_{0}^{T}\left(Y_{s}^{u, i}-\max _{j \in \mathcal{J}^{-i}}\left\{Y_{s}^{u, j}-g_{i j}\left(s, X_{s}^{t, x}\right)\right\}\right) d s=0 .
\end{array}\right.
$$

Let $s \leq T$ be fixed and $i \in \mathcal{J}$ and let $\mathcal{D}_{s}^{i}$ be the following set of admissible strategies :

$$
\mathcal{D}_{s}^{i}:=\left\{\alpha=\left(\left(\tau_{n}\right)_{n \geq 0},\left(\xi_{n}\right)_{n \geq 0}\right) \in \mathcal{D}, \xi_{0}=i, \tau_{0}=0, \tau_{1} \geq s \text { and } E\left[\left(A_{T}^{\alpha}\right)^{2}\right]<\infty\right\}
$$

where for any $r \in[0, T], A_{r}^{\alpha}$ is the cumulative switching costs up to time $r$, i.e.,

$$
A_{r}^{\alpha}:=\sum_{n \geq 1} g_{\xi_{n-1}, \xi_{n}}\left(\tau_{n}, X_{\tau_{n}}^{t, x}\right) 1_{\left[\tau_{n} \leq r\right]} \text { for } r<T \text { and } A_{T}=\lim _{r \rightarrow T} A_{r}, \mathbb{P a} \text { a.s. }
$$

Therefore and for any admissible strategy $\alpha$ we have:

$$
A_{T}^{\alpha}=\sum_{n \geq 1} g_{\xi_{n-1}, \xi_{n}}\left(\tau_{n}, X_{\tau_{n}}^{t, x}\right) 1_{\left[\tau_{n}<T\right]}
$$

Note that, by definition of the set $\mathcal{D},\left(\tau_{n}(\omega)\right)$ is a stationnary sequence (for almost all $\omega$ ) and therefore the previous sum is finite, $\mathbb{P}$-almost surely.

Let us now consider a strategy $\alpha=\left(\left(\tau_{n}\right)_{n \geq 0},\left(\xi_{n}\right)_{n \geq 0}\right) \in \mathcal{D}_{s}^{i}$ and let $\left(P^{\alpha}, Q^{\alpha}\right):=\left(P_{s}^{\alpha}, Q_{s}^{\alpha}\right)_{s \leq T}$ be the solution of the following BSDE (which is not of standard type):

$$
\left\{\begin{array}{l}
P^{\alpha} \text { is RCLL and } E\left[\sup _{s \leq T}\left|P_{s}^{\alpha}\right|^{2}\right]<\infty, Q^{\alpha} \in \mathcal{H}^{2, d} \\
P_{s}^{\alpha}=h_{\alpha}\left(X_{T}^{t, x}\right)+\int_{s}^{T} f_{\alpha}\left(r, X_{r}^{t, x}, \overrightarrow{u_{r}}, Q_{r}^{\alpha}\right) d r-\int_{s}^{T} Q_{r}^{\alpha} d B_{r}-\left(A_{T}^{\alpha}-A_{s}^{\alpha}\right), \forall s \leq T,
\end{array}\right.
$$

with

$$
h_{\alpha}(x)=\sum_{n \geq 0} h_{\xi_{n}}(x) \mathbb{1}_{\left[\tau_{n}<T \leq \tau_{n+1}[\right.} \text { and } f_{\alpha}\left(t, x,\left(\zeta^{i}\right)_{i \in \mathcal{J}}, q\right):=\sum_{n \geq 0} f_{\xi_{n}}\left(r, x,\left(\zeta^{i}\right)_{i \in \mathcal{J}}, q\right) \mathbb{1}_{\left[\tau_{n} \leq r<\tau_{n+1}[\right.}
$$

In setting up $\bar{P}^{\alpha}:=P^{\alpha}-A^{\alpha}$, we easily deduce the existence and uniqueness of the process $\left(P^{\alpha}, Q^{\alpha}\right)$, since $A^{\alpha}$ is adapted and $E\left[\left(A_{T}^{a}\right)^{2}\right]<\infty$, and the generator as well as the terminal value of the transformed BSDE are standard.

We then have the following representation for the solution of (3.10) which is the main relationship between the value function of the optimal switching problem and solutions of systems of reflected BSDEs with oblique reflection. This result usually referred as the verification result is not new and has been already shown in several contexts and under various assumptions. 
Theorem 2 Assume that for any $i, j \in \mathcal{J}$ :

(i) $f_{i}$ satisfies (H2)-(ii), (iii) and for any fixed $(\vec{y}, z)$ the mapping $(t, x) \mapsto f_{i}(t, x, \vec{y}, z)$ is $\mathcal{B}\left([0, T] \times \mathbb{R}^{k}\right)$ measurable ;

(ii) $g_{i j}\left(\right.$ resp . $\left.h_{i}\right)$ satisfies (H3) (resp. (H4)).

Then the solution of system of BSDEs (3.10) exists and satisfies:

$$
\forall s \leq T, \forall i \in \mathcal{J}, Y_{s}^{u, i}=\operatorname{ess} \sup _{\alpha \in \mathcal{D}_{s}^{i}} P_{s}^{\alpha}
$$

Thus the solution of (3.10) is unique.

Proof: Thanks to Theorem 1 and considering once more the same assumptions on the functions $\left(f_{i}, g_{i j}, h_{i}\right)_{i, j \in \mathcal{J}}$ the solution $\left(Y^{u, i}, Z^{u, i}, K^{u, i}\right)_{i \in \mathcal{J}}$ of system (3.10) exists. Next using that $Y^{u, i}$ is solution to system (3.10) and following the strategy $\alpha \in \mathcal{D}_{s}^{i}$ in (3.3), we obtain:

$$
Y_{s}^{u, i} \geq h_{\alpha}\left(X_{T}^{t, x}\right)+\int_{s}^{T} f_{\alpha}\left(r, X_{r}^{t, x}, \overrightarrow{u_{r}}, Z_{r}^{\alpha}\right) d r-\int_{s}^{T} Z_{r}^{\alpha} d B_{r}-\left(A_{T}^{\alpha}-A_{s}^{\alpha}\right)+\tilde{K}_{T}^{\alpha}
$$

where

$\tilde{K}_{r}^{a}=\left(K_{r}^{i}-K_{s}^{i}\right)$ if $t \leq r \leq \tau_{1}, \tilde{K}_{r}^{\alpha}=\tilde{K}_{\tau_{n}}^{\alpha}+\left(K_{r}^{\xi_{n}}-K_{\tau_{n}}^{\xi_{n}}\right)$ if $\tau_{n}<r \leq \tau_{n+1} ; \forall r \leq T, Z_{r}^{\alpha}=\sum_{n \geq 0} Z^{\xi_{i}} 1_{\left[\tau_{n} \leq r<\tau_{n+1}\right.}[\cdot$

As $\tilde{K}_{T}^{\alpha} \geq 0$ then we have:

$$
Y_{s}^{u, i} \geq P_{s}^{\alpha}, \forall \alpha \in \mathcal{D}_{s}^{i}
$$

Note that the right-hand side in (3.13) is not a BSDE, therefore we shall rather consider the equation satisfied by $Y^{u, i}-P^{\alpha}$ where the pair $\left(P^{\alpha}, Q^{\alpha}\right)$ satisfies (3.11). Then using an equivalent change of probability we deduce the previous inequality.

Next let $\alpha^{*}=\left(\tau_{n}^{*}, \xi_{n}^{*}\right)_{n \geq 0}$ be the strategy defined recursively as follows: $\tau_{0}^{*}=0, \xi_{0}^{*}=i$ and for $n \geq 0$,

$$
\tau_{n+1}^{*}=\inf \left\{s \geq \tau_{n}^{*}, Y_{s}^{u, \xi_{n}^{*}}=\max _{j \in \mathcal{J}^{-\xi_{n}^{*}}}\left(Y_{s}^{u, j}-g_{\xi_{n}^{*}, j}\left(s, X_{s}^{t, x}\right)\right)\right\} \wedge T
$$

and

$$
\xi_{n+1}^{*}=\operatorname{argmax}_{j \in J^{-} \xi_{n}^{*}}\left\{Y_{\tau_{n+1}^{*}}^{u, j}-g_{\xi_{n}^{*}, j}\left(\tau_{n+1}^{*}, X_{\tau_{n+1}^{*}}^{t, x}\right)\right\} .
$$

Let us show that $\alpha^{*} \in \mathcal{D}_{s}^{i}$ and, for this, let us first prove that $P\left[\tau_{n}^{*}<T, \forall n \geq 0\right]=0$. Actually assume the contrary i.e. $P\left[\tau_{n}^{*}<T, \forall n \geq 0\right]>0$. Therefore thanks to definition of $\tau_{n}^{*}$, we have:

$$
P\left[Y_{\tau_{n+1}^{*}}^{u, \xi_{n}^{*}}=Y_{\tau_{n+1}^{*}}^{u, \xi_{n+1}^{*}}-g_{\xi_{n}^{*}, \xi_{n+1}^{*}}\left(\tau_{n+1}^{*}, X_{\tau_{n+1}^{*}}^{t, x}\right), \xi_{n}^{*} \in \mathcal{J}^{-\xi_{n+1}^{*}}, \forall n \geq 1\right]>0
$$

As $\mathcal{J}$ is finite then there is a state $i_{0} \in \mathcal{J}$ and a loop $i_{0}, i_{1}, \ldots, i_{k}, i_{0}$ of elements of $\mathcal{J}$ such that $\operatorname{card}\left\{i_{0}, i_{1}, \ldots, i_{k}\right\}=k+1$ and

$$
P\left[Y_{\tau_{n+1}^{*}}^{u, i_{l}}=Y_{\tau_{n+1}^{*}}^{u, i_{l+1}}-g_{i_{l}, i_{l+1}}\left(\tau_{n+1}^{*}, X_{\tau_{n+1}^{*}}^{t, x}\right), l=0, \ldots, k,\left(i_{k+1}=i_{0}\right), \forall n \geq 1\right]>0 .
$$


Therefore taking the limit w.r.t. $n$ to obtain:

$$
P\left[Y_{\tau}^{u, i_{l}}=Y_{\tau}^{u, i_{l+1}}-g_{i_{l}, i_{l+1}}\left(\tau, X_{\tau}^{t, x}\right), l=0, \ldots, k,\left(i_{k+1}=i_{0}\right)\right]>0
$$

where $\tau:=\lim _{n \rightarrow \infty} \tau_{n}^{*}$. But this implies that

$$
P\left[g_{i_{0}, i_{1}}\left(\tau, X_{\tau}^{t, x}\right)+\cdots+g_{i_{k}, i_{0}}\left(\tau, X_{\tau}^{t, x}\right)=0\right]>0
$$

which contradicts assumption $(H 3)-(i)$. Thus we have $P\left[\tau_{n}^{*}<T, \forall n \geq 0\right]=0$.

Next it only remains to prove that $E\left[\left(A_{T}^{\alpha^{*}}\right)^{2}\right]<\infty$ and $\alpha^{*}$ is optimal in $\mathcal{D}_{s}^{i}$ for the switching problem (3.12). Actually following the strategy $\alpha^{*}$ and since $Y^{u, i}$ solves the reflected BSDE (3.10), it yields: for any $n \geq 1$,

$$
Y_{s}^{u, i}=Y_{\tau_{n}^{*}}^{u, \xi_{n}^{*}}+\int_{s}^{\tau_{n}^{*}} f_{\alpha^{*}}\left(r, X_{r}^{t, x}, \overrightarrow{u_{r}}, Z_{r}^{\alpha^{*}}\right) d r-\int_{s}^{\tau_{n}^{*}} Z_{r}^{\alpha^{*}} d B_{r}-A_{\tau_{n}^{*}}^{\alpha^{*}}
$$

noting that $K_{r}^{\xi_{n}^{*}}-K_{\tau_{n}}^{\xi_{n}^{*}}=0$ holds for any $r, \tau_{n}^{*}<r \leq \tau_{n+1}^{*}$. Taking now the limit w.r.t. $n$ in (3.14) to obtain:

$$
Y_{s}^{u, i}=h_{\alpha^{*}}\left(X_{T}^{t, x}\right)+\int_{s}^{T} f_{\alpha^{*}}\left(r, X_{r}^{t, x}, \vec{u}_{r}, Z_{r}^{\alpha^{*}}\right) d r-\int_{s}^{T} Z_{r}^{\alpha^{*}} d B_{r}-A_{T}^{\alpha^{*}} .
$$

But taking into account the assumptions (H4) and (H2)-(ii),(iii) satisfied by $h_{i}$ and $f_{i}$ respectively and since $\vec{u} \in \mathcal{H}^{2, m}$ and $Z^{a^{*}} \in \mathcal{H}^{2, d}$ and $\left(Y^{i}\right)_{i} \in\left(\mathcal{S}^{2}\right)^{m}$, we deduce from (3.15) that $E\left[\left(A_{T}^{\alpha^{*}}\right)^{2}\right]<\infty$. It follows that $\alpha^{*} \in \mathcal{D}_{s}^{i}$ and $Y_{s}^{u, i}=P_{s}^{\alpha^{*}}$, thus (3.12) holds and the solution of (3.10) is unique.

Next for $\vec{u}:=\left(u^{i}\right)_{i=1, m} \in \mathcal{H}^{2, m}$ let us define by

$$
\Phi(\vec{u}):=\left(Y^{u, i}\right)_{i=1, m}
$$

where $\left(\left(Y^{u, i}, Z^{u, i}, K^{u, i}\right)\right)_{i=1, m}$ is the solution of system (3.10) which exists and is unique if the assumptions of Theorem 2 are fulfilled. Note that when the processes $\left(Y^{u, i}\right)_{i=1, m}$ exist they belong to $\left(\mathcal{S}^{2}\right)^{m}$ and then $\Phi$ is a mapping from $\mathcal{H}^{2, m}$ to $\mathcal{H}^{2, m}$.

The following result, established by Chassagneux et al. [7], shows that $\Phi$ is a contraction in $\mathcal{H}^{2, m}$ when endowed with an appropriate equivalent norm. Therefore the existence and uniqueness of a solution for (3.3) is deduced for general functions $f_{i}$ since, contrary to Theorem 1 , they are not supposed to satisfy any monotonicity assumption. Actually we have:

Theorem 3 Assume that for any $i, j \in \mathcal{J}$ the following assumptions are fullfiled:

(i) $f_{i}$ verifies (H2)-(ii), (iii);

(ii) $g_{i j}\left(\right.$ resp. $\left.h_{i}\right)$ verifies (H3) (resp. (H4)).

Then we have:

(a) For any $\vec{u}=\left(u^{i}\right)_{i=1, m}, \vec{v}=\left(v^{i}\right)_{i=1, m} \in \mathcal{H}^{2, m}$,

$$
\forall i \in \mathcal{J}, \forall s \leq T, \mathbb{E}\left[\left|Y_{s}^{u, i}-Y_{s}^{v, i}\right|^{2}\right] \leq C\left(\|\vec{u}-\vec{v}\|_{\mathcal{H}^{2, m}}^{2}:=\mathbb{E}\left[\int_{0}^{T}\left\|\overrightarrow{u_{r}}-\overrightarrow{v_{r}}\right\|^{2} d r\right]\right) ;
$$


(b) The mapping $\Phi$ is a contraction when $\mathcal{H}^{2, m}$ is endowed with the following equivalent norm:

$$
\left\|\left(u^{i}\right)_{i=1, m}\right\|_{\beta_{0}}:=\left\{\mathbb{E}\left[\int_{0}^{T} e^{\beta_{0} s}\left(\sum_{i=1, m}\left|u_{s}^{i}\right|^{2}\right) d s\right]\right\}^{\frac{1}{2}} \text {, where } \vec{u}=\left(u^{i}\right)_{i=1, m} \in \mathcal{H}^{2, m}
$$

for some appropriate $\beta_{0} \in \mathbb{R}$.

Proof: We provide it only for the sake of completeness since it has been already given in [7]. For $i \in \mathcal{J}$, $\vec{u}$ and $\vec{v} \in \mathcal{H}^{2, m}$ let us set

$$
\varphi_{i}\left(r, X_{r}^{t, x}, z\right)=f_{i}\left(r, X_{r}^{t, x}, \overrightarrow{u_{r}}, z\right) \vee f_{i}\left(r, X_{r}^{t, x}, \overrightarrow{v_{r}}, z\right), r \leq T
$$

and let us consider the solution, denoted by $\left(\tilde{Y}^{i}, \tilde{Z}^{i}, \tilde{K}^{i}\right)_{i \in \mathcal{J}}$, of the system of obliquely reflected BSDEs associated with $\left(\varphi_{i}\left(r, X_{r}^{t, x}, z\right)\right)_{i \in \mathcal{J}},\left(h_{i}\right)_{i \in \mathcal{J}}$ and $\left(g_{i j}\right)_{i, j \in \mathcal{J}}$ which exists and is unique by Theorem 1 , As shown in Theorem 2, the following representation holds true:

$$
\forall s \leq T, \tilde{Y}_{s}^{i}=\operatorname{ess} \sup _{a \in \mathcal{D}_{s}^{i}} \tilde{P}_{s}
$$

where for $a \in \mathcal{D}_{s}^{i}$ the pair of processes $\left(\tilde{P}^{a}, \tilde{Q}^{a}\right)$ verifies:

$$
\left\{\begin{array}{l}
\tilde{P}^{a} \text { is RCLL and } E\left[\sup _{\eta \leq T}\left|\tilde{P}_{\eta}^{a}\right|^{2}\right]<\infty, \tilde{Q}^{a} \in \mathcal{H}^{2, d} ; \\
\tilde{P}_{\eta}^{a}=h_{a}\left(X_{T}^{t, x}\right)+\int_{\eta}^{T} \varphi_{a}\left(r, X_{r}^{t, x}, \tilde{Q}_{r}^{a}\right) d r-\int_{\eta}^{T} \tilde{Q}_{r}^{a} d B_{r}-\left(A_{T}^{a}-A_{\eta}^{a}\right), \forall \eta \leq T .
\end{array}\right.
$$

Additionally an optimal strategy $\tilde{a}$ exists i.e. $\tilde{Y}_{s}^{i}=\tilde{P}_{s}^{\tilde{a}}$. Note here that the dependence of $P_{s}^{\tilde{a}}$ on $i$ is made through the strategy $\tilde{a}$ which belongs to $\mathcal{D}_{s}^{i}$. Now since for any $r \leq T$ and $z \in \mathbb{R}^{d}, \varphi_{i}\left(r, X_{r}^{t, x}, z\right) \geq$ $f_{i}\left(r, X_{r}^{t, x}, \overrightarrow{u_{r}}, z\right)$ and $\varphi_{i}\left(r, X_{r}^{t, x}, z\right) \geq f_{i}\left(r, X_{r}^{t, x}, \overrightarrow{v_{r}}, z\right)$ then by comparison and uniqueness (see Remark 1) we have:

$$
Y^{u, i} \leq \tilde{Y}^{i} \text { and } Y^{v, i} \leq \tilde{Y}^{i}
$$

Next for $a \in \mathcal{D}_{s}^{i}$, let $\left(P_{r}^{a}, Q_{r}^{a}\right)_{r \leq T}$ be the solution of the non-standard BSDE (3.11) and let $\left(P_{r}^{\prime a}, Q_{r}^{\prime a}\right)_{r \leq T}$ be the solution of the same non-standard BSDE with generator $f_{a}\left(r, X_{r}^{t, x}, \overrightarrow{v_{r}}, z\right)$. Then we have:

$$
P_{s}^{\tilde{a}} \leq Y_{s}^{u, i} \leq \tilde{Y}_{s}^{i}=\tilde{P}_{s}^{\tilde{a}} \text { and } P_{s}^{(\tilde{a}} \leq Y_{s}^{v, i} \leq \tilde{Y}_{s}^{i}=\tilde{P}_{s}^{\tilde{a}}
$$

which implies,

$$
\left|Y_{s}^{u, i}-Y_{s}^{v, i}\right| \leq\left|\tilde{P}_{s}^{\tilde{a}}-P_{s}^{\tilde{a}}\right|+\left|\tilde{P}_{s}^{\tilde{a}}-P_{s}^{\prime \tilde{a}}\right|
$$

But for any $\eta \leq T$ we have:

$$
\begin{aligned}
& \tilde{P}_{\eta}^{\tilde{a}}-P_{\eta}^{\tilde{a}}= \\
& \int_{\eta}^{T}\left\{f_{\tilde{a}}\left(r, X_{r}^{t, x}, \overrightarrow{u_{r}}, \tilde{Q}_{r}^{\tilde{a}}\right) \vee f_{\tilde{a}}\left(r, X_{r}^{t, x}, \overrightarrow{v_{r}}, \tilde{Q}_{r}^{\tilde{a}}\right)-f_{\tilde{a}}\left(r, X_{r}^{t, x}, \overrightarrow{u_{r}}, Q_{r}^{\tilde{a}}\right)\right\} d r-\int_{\eta}^{T}\left\{\tilde{Q}_{r}^{\tilde{a}}-Q_{r}^{\tilde{a}}\right\} d B_{r},
\end{aligned}
$$

and a similar equation is valid for $\tilde{P}_{\eta}^{\tilde{a}}-P_{\eta}^{\prime \tilde{a}}$. Next using Itô's formula to obtain:

$$
\begin{aligned}
\left|\tilde{P}_{\eta}^{\tilde{a}}-P_{\eta}^{\tilde{a}}\right|^{2}+\int_{\eta}^{T}\left|\tilde{Q}_{r}^{\tilde{a}}-Q_{r}^{\tilde{a}}\right|^{2} d r=-2 \int_{\eta}^{T}\left(\tilde{P}_{r}^{\tilde{a}}-P_{r}^{\tilde{a}}\right)\left\{\tilde{Q}_{r}^{\tilde{a}}-Q_{r}^{\tilde{a}}\right\} d B_{r} \\
\quad+2 \int_{\eta}^{T}\left(\tilde{P}_{r}^{\tilde{a}}-P_{r}^{\tilde{a}}\right)\left\{f_{\tilde{a}}\left(r, X_{r}^{t, x}, \overrightarrow{u_{r}}, \tilde{Q}_{r}^{\tilde{a}}\right) \vee f_{\tilde{a}}\left(r, X_{r}^{t, x}, \overrightarrow{v_{r}}, \tilde{Q}_{r}^{\tilde{a}}\right)-f_{\tilde{a}}\left(r, X_{r}^{t, x}, \overrightarrow{u_{r}}, Q_{r}^{\tilde{a}}\right)\right\} d r .
\end{aligned}
$$


As for any $x, y \in \mathbb{R}$ we have $|x \vee y-y| \leq|x-y|$, then

$$
\begin{aligned}
\left|\tilde{P}_{\eta}^{\tilde{a}}-P_{\eta}^{\tilde{a}}\right|^{2}+\int_{\eta}^{T}\left|\tilde{Q}_{r}^{\tilde{a}}-Q_{r}^{\tilde{a}}\right|^{2} d r=-2 \int_{\eta}^{T}\left(\tilde{P}_{r}^{\tilde{a}}-P_{r}^{\tilde{a}}\right)\left\{\tilde{Q}_{r}^{\tilde{a}}-Q_{r}^{\tilde{a}}\right\} d B_{r} \\
\quad+2 \int_{\eta}^{T}\left|\tilde{P}_{r}^{\tilde{a}}-P_{r}^{\tilde{a}}\right|\left|f_{\tilde{a}}\left(r, X_{r}^{t, x}, \overrightarrow{v_{r}}, \tilde{Q}_{r}^{\tilde{a}}\right)-f_{\tilde{a}}\left(r, X_{r}^{t, x}, \overrightarrow{u_{r}}, Q_{r}^{\tilde{a}}\right)\right| d r
\end{aligned}
$$

Now classically we obtain the existence of a real constant $C \geq 0$ such that:

$$
\mathbb{E}\left[\sup _{\eta \leq T}\left|\tilde{P}_{\eta}^{\tilde{a}}-P_{\eta}^{\tilde{a}}\right|^{2}\right] \leq C \mathbb{E}\left[\int_{0}^{T}\left\|\overrightarrow{u_{r}}-\overrightarrow{v_{r}}\right\|^{2} d r\right]
$$

In the same way considering $\left|\tilde{P}_{\eta}^{\tilde{a}}-P_{\eta}^{\prime \tilde{a}}\right|^{2}$ we obtain a similar inequality as $(3.20)$ where $P^{\tilde{a}}$ is replaced by $P^{\prime a \tilde{a}}$. Finally going back to (3.19), squarring and taking the expectation, we obtain the first estimate.

Let us now show that $\Phi$ is a contraction. Let $\beta>0$ and let us make use of Itô's formula to obtain:

$$
\begin{aligned}
e^{\beta \eta} \mid \tilde{P}_{\eta}^{\tilde{a}}- & \left.P_{\eta}^{\tilde{a}}\right|^{2}+\int_{\eta}^{T} e^{\beta r}\left|\tilde{Q}_{r}^{\tilde{a}}-Q_{r}^{\tilde{a}}\right|^{2} d r=-2 \int_{\eta}^{T} e^{\beta r}\left(\tilde{P}_{r}^{\tilde{a}}-P_{r}^{\tilde{a}}\right)\left(\tilde{Q}_{r}^{\tilde{a}}-Q_{r}^{\tilde{a}}\right) d B_{r}-\beta \int_{\eta}^{T} e^{\beta r}\left|\tilde{P}_{r}^{\tilde{a}}-P_{r}^{\tilde{a}}\right|^{2} d r \\
& +2 \int_{\eta}^{T} e^{\beta r}\left(\tilde{P}_{r}^{\tilde{a}}-P_{r}^{\tilde{a}}\right)\left\{f_{\tilde{a}}\left(r, X_{r}^{t, x}, \overrightarrow{u_{r}}, \tilde{Q}_{r}^{\tilde{a}}\right) \vee f_{\tilde{a}}\left(r, X_{r}^{t, x}, \overrightarrow{v_{r}}, \tilde{Q}_{r}^{\tilde{a}}\right)-f_{\tilde{a}}\left(r, X_{r}^{t, x}, \overrightarrow{u_{r}}, Q_{r}^{\tilde{a}}\right)\right\} d r, \eta \leq T .
\end{aligned}
$$

But

$$
\mid f_{\tilde{a}}\left(r, X_{r}^{t, x}, \overrightarrow{u_{r}}, \tilde{Q}_{r}^{\tilde{a}}\right) \vee f_{\tilde{a}}\left(r, X_{r}^{t, x}, \overrightarrow{v_{r}}, \tilde{Q}_{r}^{\tilde{a}}\right)-f_{\tilde{a}}\left(r, X_{r}^{t, x}, \overrightarrow{u_{r}}, Q_{r}^{\tilde{a}} \mid \leq C\left(\left|\overrightarrow{v_{r}}-\overrightarrow{u_{r}}\right|+\left|\tilde{Q}_{r}^{\tilde{a}}-Q_{r}^{\tilde{a}}\right|\right)\right.
$$

where $C$ is the Lipschitz constant of $f$. Therefore taking expectation in the previous equation and using both inequalities $2 C x y \leq(C x)^{2}+y^{2}$ and $2 x y \leq \frac{x^{2}}{\sqrt{\beta}}+\sqrt{\beta} y^{2}$ for any $x, y \in \mathbb{R}$, we obtain: $\forall \eta \leq T$,

$$
\mathbb{E}\left[e^{\beta \eta}\left|\tilde{P}_{\eta}^{\tilde{a}}-P_{\eta}^{\tilde{a}}\right|^{2}\right] \leq\left(C^{2}+C \sqrt{\beta}-\beta\right) \mathbb{E}\left[\int_{\eta}^{T} e^{\beta r}\left|\tilde{P}_{r}^{\tilde{a}}-P_{r}^{\tilde{a}}\right|^{2} d r\right]+\frac{C}{\sqrt{\beta}} \mathbb{E}\left[\int_{\eta}^{T} \| \overrightarrow{u_{r}}-\left.\overrightarrow{v_{r}}\right|^{2} d r\right]
$$

But the same estimate can be obtained for $\mathbb{E}\left[e^{\beta \eta}\left|\tilde{P}_{\eta}^{\tilde{a}}-P_{\eta}^{\prime \tilde{a}}\right|^{2}\right]$. Taking $\beta \geq C^{2}+C \sqrt{\beta}$ and going back to (3.19) to obtain:

$$
\mathbb{E}\left[e^{\beta s}\left|Y_{s}^{u, i}-Y_{s}^{v, i}\right|^{2}\right] \leq \frac{2 C}{\sqrt{\beta}} \mathbb{E}\left[\int_{0}^{T} \| \overrightarrow{u_{r}}-\left.\overrightarrow{v_{r}}\right|^{2} d r\right] .
$$

Next summing for $i=1, m$ in (3.21) and integrating w.r.t. $d t$ we obtain that:

$$
\|\Phi(\vec{u})-\Phi(\vec{v})\|_{\beta}^{2}:=\mathbb{E}\left[\int_{0}^{T} e^{\beta s}\left(\sum_{i=1, m}\left|Y_{s}^{u, i}-Y_{s}^{v, i}\right|^{2}\right) d s\right] \leq \frac{2 C T}{\sqrt{\beta}} \mathbb{E}\left[\int_{0}^{T}\left\|\overrightarrow{u_{r}}-\overrightarrow{v_{r}}\right\|^{2} d r\right] .
$$

Choosing now $\beta=\beta_{0} \geq 2 \max \left\{(2 C T)^{2}, C^{2}+C \sqrt{\beta}\right\}$ yields that $\Phi$ is a contraction in the Banach space $\left(\mathcal{H}^{2, m},\|\cdot\|_{\beta_{0}}\right)$, therefore it has a fixed point $\left(Y^{i}\right)_{i=1, m}$ which can be chosen continuous since $\Phi\left(\left(Y^{i}\right)_{i=1, m}\right) \in\left(\mathcal{S}^{2}\right)^{m}$. Thus the system of reflected BSDEs with interconnected obstacles has a unique solution.

Remark 3 Let $\left(Y^{i, 0}\right)_{i \in \mathcal{J}}$ be fixed processes of $\mathcal{H}^{2, m}$ and for $n \geq 1$ let us $\operatorname{set}\left(Y^{i, n}\right)_{i \in \mathcal{J}}=\Phi\left(\left(Y^{i, n-1}\right)_{i \in \mathcal{J}}\right)$. Then the sequence $\left(\left(Y^{i, n}\right)_{i \in \mathcal{J}}\right)_{n \geq 0}$ converges in $\left(\mathcal{H}^{2, m},\|\|.\right)$ to the unique solution of the system of reflected BSDEs associated with $\left(\left(f_{i}\right)_{i \in \mathcal{J}},\left(g_{i j}\right)_{i, j \in \mathcal{J}},\left(h_{i}\right)_{i \in \mathcal{J}}\right)$ since $\Phi$ is a contraction in $\left(\mathcal{H}^{2, m},\|\cdot\|_{\beta_{0}}\right)$ and the norms $\|\cdot\|_{\beta_{0}}$ and $\|$.$\| are equivalent.$ 


\section{Uniqueness of the solution of the system of PDEs}

In this section we deal with the issue of uniqueness of the solution of system (2.3) and to do so, we first establish an auxiliary result which is a classical one in viscosity literature (see e.g. [26], pp. 76).

Lemma 4.1 Let $\left(v_{i}(t, x)\right)_{i=1, m}$ be a supersolution of the system (2.3), then for any $\gamma \geq 0$ there exists $\lambda_{0}>0$ which does not depend on $\theta$ such that for any $\lambda \geq \lambda_{0}$ and $\theta>0$, the m-uplet $\left(v_{i}(t, x)+\right.$ $\left.\theta e^{-\lambda t}|x|^{2 \gamma+2}\right)_{i=1, m}$ is a supersolution for (2.3).

Proof: Without loss of generality we assume that the functions $v_{1}, \ldots, v_{m}$ are $l s c$. For sake of convenience, we do not use the previous definition of a supersolution but an equivalent one (see e.g. [8]). Let $i \in \mathcal{J}$ be fixed and let $\varphi \in \mathcal{C}^{1,2}$ be such that the function $\varphi-\left(v^{i}+\theta e^{-\lambda t}|x|^{2 \gamma+2}\right)$ has a local maximum in $(t, x)$ which is equal to 0 . As $\left(v_{i}\right)_{i=1, m}$ is a supersolution for (2.3), then we have: $\forall i \in \mathcal{J}$,

$$
\begin{aligned}
& \min \left\{v_{i}(t, x)-\max _{j \in \mathcal{J}^{-i}}\left(-g_{i j}(t, x)+v_{j}(t, x)\right)\right. \\
& \quad-\partial_{t}\left(\varphi(t, x)-\theta e^{-\lambda t}|x|^{2 \gamma+2}\right)-\frac{1}{2} \operatorname{Tr}\left(\sigma \cdot \sigma^{\top}(t, x) D_{x x}^{2}\left(\varphi(t, x)-\theta e^{-\lambda t}|x|^{2 \gamma+2}\right)\right) \\
& \left.-b(t, x)^{\top} \cdot D_{x}\left(\varphi(t, x)-\theta e^{-\lambda t}|x|^{2 \gamma+2}\right)-f_{i}\left(t, x,\left(v^{1}, \ldots, v^{m}\right)(t, x), \sigma^{\top}(t, x) D_{x}\left(\varphi(t, x)-\theta e^{-\lambda t}|x|^{2 \gamma+2}\right)\right)\right\} \geq 0
\end{aligned}
$$

which implies that

$$
\begin{aligned}
\left(v_{i}(t, x)+\right. & \left.\theta e^{-\lambda t}|x|^{2 \gamma+2}\right)-\max _{j \in \mathcal{J}^{-i}}\left(-g_{i j}(t, x)+\left(v_{j}(t, x)+\theta e^{-\lambda t}|x|^{2 \gamma+2}\right)\right) \\
& =v_{i}(t, x)-\max _{j \in \mathcal{J}^{-i}}\left(-g_{i j}(t, x)+v_{j}(t, x)\right) \geq 0 .
\end{aligned}
$$

On the other hand:

$$
\begin{aligned}
& -\partial_{t}\left(\varphi(t, x)-\theta e^{-\lambda t}|x|^{2 \gamma+2}\right)-\frac{1}{2} \operatorname{Tr}\left(\sigma \cdot \sigma^{\top}(t, x) D_{x x}^{2}\left(\varphi(t, x)-\theta e^{-\lambda t}|x|^{2 \gamma+2}\right)\right) \\
& -D_{x}\left(\varphi(t, x)-\theta e^{-\lambda t}|x|^{2 \gamma+2}\right) \cdot b(t, x)-f_{i}\left(t, x,\left(v^{1}, \ldots, v^{m}\right)(t, x), \sigma^{\top}(t, x) D_{x}\left(\varphi(t, x)-\theta e^{-\lambda t}|x|^{2 \gamma+2}\right)\right) \geq 0
\end{aligned}
$$

and then

$$
\begin{array}{r}
-\partial_{t} \varphi(t, x)-\frac{1}{2} \operatorname{Tr}\left(\sigma \cdot \sigma^{\top}(t, x) D_{x x}^{2} \varphi(t, x)\right)-b(t, x)^{\top} \cdot D_{x} \varphi(t, x) \\
-f_{i}\left(t, x,\left(v^{i}(t, x)+\theta e^{-\lambda t}|x|^{2 \gamma+2}\right)_{i=1, m}, \sigma^{\top}(t, x) D_{x} \varphi(t, x)\right) \\
\geq \theta \lambda e^{-\lambda t}|x|^{2 \gamma+2}-\frac{1}{2} \theta e^{-\lambda t} \operatorname{Tr}\left(\sigma \cdot \sigma^{\top}(t, x) D_{x x}^{2}|x|^{2 \gamma+2}\right)-\theta e^{-\lambda t} D_{x}\left(|x|^{2 \gamma+2}\right) . b(t, x) \\
+\left[f_{i}\left(t, x,\left(v^{1}, \ldots, v^{m}\right)(t, x), \sigma^{\top}(t, x) D_{x}\left(\varphi(t, x)-\theta e^{-\lambda t}|x|^{2 \gamma+2}\right)\right)\right. \\
\left.-f_{i}\left(t, x,\left(v^{1}, \ldots, v^{m}\right)(t, x), \sigma^{\top}(t, x) D_{x} \varphi(t, x)\right)\right] .
\end{array}
$$

But the last term in the right-hand side of this latter inequality is equal to

$$
\theta e^{-\lambda t} C_{t, x, \theta, \lambda}^{i} \cdot \sigma^{\top}(t, x) D_{x}\left(|x|^{2 \gamma+2}\right)
$$

where $C_{t, x, \theta, \lambda}^{i}$ is bounded by a constant independent of $\theta$ since the function $f_{i}$ is uniformly Lipschitz w.r.t. $z$. Therefore, taking into account the growth conditions on $b$ and $\sigma$, there exists a constant $\lambda_{0} \in \mathbb{R}^{+}$which does not depend on $\theta$ such that if $\lambda \geq \lambda_{0}$, the right-hand side of (4.2) is non-negative. 
Henceforth, noting that $i$ is arbitrary in $\mathcal{J}$ together with (4.1), we obtain that $\left(v^{i}+\theta e^{-\lambda t}|x|^{2 \gamma+2}\right)_{i=1, m}$ is a viscosity supersolution for (2.3).

We now establish the comparison property between supersolutions and subsolutions of (2.3) in the case when $f_{i}$ does not depend on $\left(y^{1}, \ldots, y^{i-1}, y^{i+1}, \ldots, y^{m}\right)$ for any $i \in \mathcal{J}$. Actually let us introduce the following assumption on the functions $f_{i}$ 's.

[H5]: For any $i \in \mathcal{J}$, the function $f_{i}$ does not depend on $\left(y^{1}, \ldots, y^{i-1}, y^{i+1}, \ldots, y^{m}\right)$. Note that this assumption replaces assumption (H2)(iv): this last one does not make sense any more when $f_{i}$ depends only on $y^{i}, z^{i}$.

Proposition 4.1 Assume both (H3) and (H4) and let suppose that the functions $f_{i}, i \in \mathcal{J}$, verify (H2)(i), (ii) and (H5). Let $\left(u^{i}(t, x)\right)_{i=1, m}\left(\right.$ resp. $\left.\left(v^{i}(t, x)\right)_{i=1, m}\right)$ be a subsolution (resp. a supersolution) of the system (2.3) which belongs to $\Pi^{g}$, then for any $i \in \mathcal{J}$, we have:

$$
\forall(t, x) \in[0, T] \times \mathbb{R}^{k}, u^{i}(t, x) \leq v^{i}(t, x)
$$

Proof: First w.l.o.g we assume that $u_{i}\left(\right.$ resp. $\left.w^{i}\right)$ is $u s c$ (resp. lsc) for any $i \in \mathcal{J}$. Next let $\gamma>0$ and $C$ be such that that for any $i \in \mathcal{J}$ we have:

$$
\left|u_{i}(t, x)\right|+\left|v^{i}(t, x)\right| \leq C\left(1+|x|^{\gamma}\right), \forall(t, x) \in[0, T] \times \mathbb{R}^{k}
$$

For sake of clarity, the proof is divided into two steps.

$\underline{\text { Step 1: }}$ To begin with we additionally assume that the functions $f_{i}, i \in \mathcal{J}$, satisfy:

$$
\exists \lambda<0 \text { s.t. } \forall t, x, z, \forall u \geq v, f_{i}(t, x, u, z)-f_{i}(t, x, v, z) \leq \lambda(u-v) .
$$

According to the previous lemma we know that for any $\theta>0$ and $\lambda$ large enough $\left(v_{i}(t, x)+\right.$ $\left.\theta e^{-\lambda t}|x|^{2 \gamma+2}\right)_{i=1, m}$ is also a supersolution for (2.3). Therefore it is enough to show that for any $i \in \mathcal{J}$, we have:

$$
\forall(t, x) \in[0, T] \times \mathbb{R}^{k}, u^{i}(t, x) \leq v^{i}(t, x)+\theta e^{-\lambda t}|x|^{2 \gamma+2},
$$

since in taking the limit as $\theta \rightarrow 0$ we obtain the desired result. So let us set $w^{i, \theta, \lambda}(t, x)=v^{i}(t, x)+$ $\theta e^{-\lambda t}|x|^{2 \gamma+2},(t, x) \in[0, T] \times \mathbb{R}^{k}$ and we still denote $w^{i, \theta, \lambda}$ by $w^{i}$. Next assume there exists a point $(\bar{t}, \bar{x}) \in[0, T] \times \mathbb{R}^{k}$ such that for $i \in \mathcal{J}: \max _{i \in \mathcal{J}}\left(u^{i}(\bar{t}, \bar{x})-w^{i}(\bar{t}, \bar{x})\right)>0$. Next using the growth condition there exists $R>0$ such that:

$$
\forall(t, x) \in[0, T] \times \mathbb{R}^{k} \text { s.t. }|x| \geq R, u^{i}(t, x)-w^{i}(t, x)<0
$$


Taking into account the values of the subsolution and the supersolution at $T$, it implies that

$$
\begin{aligned}
& 0<\max _{(t, x) \in[0, T] \times \mathbb{R}^{k}} \max _{i \in \mathcal{J}}\left(u^{i}(t, x)-w^{i}(t, x)\right)= \\
& \quad \max _{(t, x) \in[0, T[\times B(0, R)} \max _{i \in \mathcal{J}}\left(u^{i}(t, x)-w^{i}(t, x)\right)=\max _{i \in \mathcal{J}}\left(u^{i}\left(t^{*}, x^{*}\right)-w^{i}\left(t^{*}, x^{*}\right)\right),
\end{aligned}
$$

where $B(0, R)$ is the open ball in $\mathbb{R}^{k}$ centered in 0 and of radius $R$ and $\left(t^{*}, x^{*}\right) \in[0, T[\times B(0, R)$.

Now let us define $\tilde{\mathcal{J}}$ as:

$$
\tilde{\mathcal{J}}:=\left\{j \in \mathcal{J}, u_{j}\left(t^{*}, x^{*}\right)-w^{j}\left(t^{*}, x^{*}\right)=\max _{k \in \mathcal{J}}\left(u_{k}\left(t^{*}, x^{*}\right)-w^{k}\left(t^{*}, x^{*}\right)\right)\right\} .
$$

First note that $\tilde{\mathcal{J}}$ is not empty. Next for $j \in \tilde{\mathcal{J}}$ and $n \geq 1$, let us define:

$$
\Phi_{n}^{j}(t, x, y):=u_{j}(t, x)-w^{j}(t, y)-\varphi_{n}(t, x, y),(t, x, y) \in[0, T] \times \mathbb{R}^{2 k}
$$

where: $\varphi_{n}(t, x, y):=n|x-y|^{2 \gamma+2}+\left|x-x^{*}\right|^{2}+\left|t-t^{*}\right|^{2}$. Now let $\left(t_{n}, x_{n}, y_{n}\right) \in[0, T] \times B^{\prime}(0, R)^{2}$ be such that

$$
\Phi_{n}^{j}\left(t_{n}, x_{n}, y_{n}\right)=\max _{(t, x, y) \in[0, T] \times B^{\prime}(0, R)^{2}} \Phi_{n}^{j}(t, x, y),
$$

which exists since $\Phi_{n}^{j}$ is usc $\left(B^{\prime}(0, R)\right.$ is the closure of $\left.B(0, R)\right)$. Then we have:

$\Phi_{n}^{j}\left(t^{*}, x^{*}, x^{*}\right)=u_{j}\left(t^{*}, x^{*}\right)-w^{j}\left(t^{*}, x^{*}\right) \leq u_{j}\left(t^{*}, x^{*}\right)-w^{j}\left(t^{*}, x^{*}\right)+\varphi_{n}\left(t_{n}, x_{n}, y_{n}\right) \leq u_{j}\left(t_{n}, x_{n}\right)-w^{j}\left(t_{n}, y_{n}\right)$.

The definition of $\varphi_{n}$ together with the growth condition of $u_{j}$ and $w^{j}$ implies that $\left(x_{n}-y_{n}\right)_{n \geq 1}$ converges to 0 . Next for any subsequence $\left(\left(t_{n_{l}}, x_{n_{l}}, y_{n_{l}}\right)\right)_{l}$ which converges to $(\tilde{t}, \tilde{x}, \tilde{x})$ we deduce from (4.7) that

$$
u_{j}\left(t^{*}, x^{*}\right)-w^{j}\left(t^{*}, x^{*}\right) \leq u_{j}(\tilde{t}, \tilde{x})-w^{j}(\tilde{t}, \tilde{x})
$$

since $u_{j}$ is $u s c$ and $w^{j}$ is $l s c$. As the maximum of $u_{j}-w^{j}$ on $[0, T] \times B^{\prime}(0, R)$ is reached in $\left(t^{*}, x^{*}\right)$ then this last inequality is actually an equality. It implies, from the definition of $\varphi_{n}$ and (4.7), that the sequence $\left(\left(t_{n}, x_{n}, y_{n}\right)\right)_{n}$ converges to $\left(t^{*}, x^{*}, x^{*}\right)$ from which we deduce

$$
n\left|x_{n}-y_{n}\right|^{2 \gamma+2} \rightarrow_{n} 0 \text { and }\left(u_{j}\left(t_{n}, x_{n}\right), w^{j}\left(t_{n}, y_{n}\right)\right) \rightarrow_{n}\left(u_{j}\left(t^{*}, x^{*}\right), w^{j}\left(t^{*}, y^{*}\right)\right)
$$

Actually this latter convergence holds since from (4.7) we first obtain,

$$
u_{j}\left(t^{*}, x^{*}\right)-w^{j}\left(t^{*}, x^{*}\right) \leq \frac{\lim }{n} u_{j}\left(t_{n}, x_{n}\right)-\varlimsup_{n} w^{j}\left(t_{n}, y_{n}\right)
$$

whereas the fact that $u_{j}$ (resp. $w_{j}$ ) is usc (resp. lsc) gives

$$
\varlimsup_{n} u_{j}\left(t_{n}, x_{n}\right)-\varliminf_{n} w^{j}\left(t_{n}, y_{n}\right) \leq u_{j}\left(t^{*}, x^{*}\right)-w^{j}\left(t^{*}, x^{*}\right) .
$$

All these inequalities imply that

$$
\frac{\varliminf_{n}}{n} u_{j}\left(t_{n}, x_{n}\right)=\varlimsup_{n} u_{j}\left(t_{n}, x_{n}\right)=u_{j}\left(t^{*}, x^{*}\right) \text { and } \varliminf_{n} w^{j}\left(t_{n}, x_{n}\right)=\varlimsup_{n} w^{j}\left(t_{n}, x_{n}\right)=w^{j}\left(t^{*}, x^{*}\right)
$$


Next as in [21, let us show by contradiction that for some $k \in \tilde{\mathcal{J}}$ we have:

$$
u_{k}\left(t^{*}, x^{*}\right)>\max _{j \in \mathcal{J}^{-k}}\left(u_{j}\left(t^{*}, x^{*}\right)-g_{k j}\left(t^{*}, x^{*}\right)\right)
$$

Actually suppose that for any $k \in \tilde{\mathcal{J}}$ we have:

$$
u_{k}\left(t^{*}, x^{*}\right) \leq \max _{j \in \mathcal{J}^{-k}}\left(u_{j}\left(t^{*}, x^{*}\right)-g_{k j}\left(t^{*}, x^{*}\right)\right)
$$

then there exists $j \in \mathcal{J}^{-k}$ such that

$$
u_{k}\left(t^{*}, x^{*}\right)-u_{j}\left(t^{*}, x^{*}\right) \leq-g_{k j}\left(t^{*}, x^{*}\right) .
$$

But $w^{k}$ is a supersolution of (2.3), therefore we have

$$
w^{k}\left(t^{*}, x^{*}\right) \geq w^{j}\left(t^{*}, x^{*}\right)-g_{k j}\left(t^{*}, x^{*}\right)
$$

and then

$$
u_{k}\left(t^{*}, x^{*}\right)-u_{j}\left(t^{*}, x^{*}\right) \leq-g_{k j}\left(t^{*}, x^{*}\right) \leq w^{k}\left(t^{*}, x^{*}\right)-w^{j}\left(t^{*}, x^{*}\right)
$$

Therefore

$$
u_{k}\left(t^{*}, x^{*}\right)-w^{k}\left(t^{*}, x^{*}\right)=u_{j}\left(t^{*}, x^{*}\right)-w^{j}\left(t^{*}, x^{*}\right)
$$

which implies that $j$ also belongs to $\tilde{\mathcal{J}}$ and

$$
u_{k}\left(t^{*}, x^{*}\right)-u_{j}\left(t^{*}, x^{*}\right)=-g_{k j}\left(t^{*}, x^{*}\right) .
$$

Repeating this procedure as many times as necessary and since $\tilde{\mathcal{J}}$ is finite we get the existence of a loop of indices $i_{1}, \ldots, i_{p}, i_{p+1}$ of $\tilde{\mathcal{J}}$ such that $i_{1}=i_{p+1}$ and

$$
g_{i_{1}, i_{2}}\left(t^{*}, x^{*}\right)+\cdots+g_{i_{p}, i_{p+1}}\left(t^{*}, x^{*}\right)=0 .
$$

But this contradicts the assumption (H3) on $g_{i j}, i, j \in \mathcal{J}$, whence the desired result.

To proceed let us consider $k \in \tilde{\mathcal{J}}$ such that:

$$
u_{k}\left(t^{*}, x^{*}\right)>\max _{j \in \mathcal{J}^{-k}}\left(u_{j}\left(t^{*}, x^{*}\right)-g_{k j}\left(t^{*}, x^{*}\right)\right) .
$$

As the functions $u_{j}, j \in \mathcal{J}$, are $u s c$ and $g_{i j}$ are continuous, then there exists $\rho>0$ such that for $(t, x) \in B\left(\left(t^{*}, x^{*}\right), \rho\right)$ we have $u_{k}(t, x)>\max _{j \in \mathcal{J}^{-k}}\left(u_{j}(t, x)-g_{k j}(t, x)\right)$. Next and by construction it holds that $\left(t_{n}, x_{n}, u_{k}\left(t_{n}, x_{n}\right)\right)_{n} \rightarrow_{n}\left(t^{*}, x^{*}, u_{k}\left(t^{*}, x^{*}\right)\right)$ and once more since $u_{j}$ is usc then for $n$ large enough we have:

$$
u_{k}\left(t_{n}, x_{n}\right)>\max _{j \in \mathcal{J}^{-k}}\left(u_{j}\left(t_{n}, x_{n}\right)-g_{k j}\left(t_{n}, x_{n}\right)\right)
$$


Now applying Crandall-Ishii-Lions's Lemma (see e.g. [8] or [14], pp.216) with $\Phi_{n}^{k}$ (note that $k \in \tilde{\mathcal{J}}$ and (4.9) is satisfied) in $\left(t_{n}, x_{n}, y_{n}\right)$, there exist $\left(p_{u}^{n}, q_{u}^{n}, M_{u}^{n}\right) \in \bar{J}^{2,+} u_{k}\left(t_{n}, x_{n}\right)$ and $\left(p_{w}^{n}, q_{w}^{n}, M_{w}^{n}\right) \in$ $\bar{J}^{2,-} w^{k}\left(t_{n}, y_{n}\right)$ such that:

$$
\begin{gathered}
p_{u}^{n}-p_{w}^{n}=\partial_{t} \varphi_{n}\left(t_{n}, x_{n}, y_{n}\right), q_{u}^{n}\left(\operatorname{resp.} q_{w}^{n}\right)=\partial_{x} \varphi_{n}\left(t_{n}, x_{n}, y_{n}\right)\left(\text { resp. }=-\partial_{y} \varphi_{n}\left(t_{n}, x_{n}, y_{n}\right)\right) \text { and } \\
\left(\begin{array}{cc}
M_{u}^{n} & 0 \\
0 & -N_{w}^{n}
\end{array}\right) \leq A_{n}+\frac{1}{2 n} A_{n}^{2}
\end{gathered}
$$

where $A_{n}=D_{(x, y)}^{2} \varphi_{n}\left(t_{n}, x_{n}, y_{n}\right)$. But

$$
\begin{gathered}
\partial_{t} \varphi_{n} k(t, x, y)=2\left(t-t^{*}\right), \quad \partial_{x} \varphi_{n}(t, x, y)=2(\gamma+1) n(x-y)|x-y|^{2 \gamma}+2\left(x-x^{*}\right) \text { and } \\
\partial_{y} \varphi_{n}(t, x, y)=2 n(\gamma+1)(x-y)|x-y|^{2 \gamma}
\end{gathered}
$$

On the other hand,

$$
D_{(x, y)}^{2} \varphi(t, x, y)=\left(\begin{array}{ll}
D_{x x}^{2} \varphi\left(t_{n}, x_{n}, y_{n}\right) & D_{x y}^{2} \tilde{\varphi}(t, x, y) \\
D_{x y}^{2} \varphi(t, x, y) & D_{y y}^{2} \varphi(t, x, y)
\end{array}\right)
$$

with

$$
\begin{gathered}
D_{x x}^{2} \varphi(t, x, y)=2 n(\gamma+1)|x-y|^{2 \gamma-2}\left\{|x-y|^{2} I_{k}+2 \gamma(x-y)(x-y)^{\top}\right\}+2 I_{k}, \\
D_{y y}^{2} \varphi(t, x, y)=2 n(\gamma+1)|x-y|^{2 \gamma-2}\left\{|x-y|^{2} I_{k}+2 \gamma(x-y)(x-y)^{\top}\right\}
\end{gathered}
$$

and finally

$$
D_{x y}^{2} \varphi(t, x, y)=-2 n(\gamma+1)|x-y|^{2 \gamma} I_{k}-4 n \gamma(\gamma+1)(x-y)(x-y)^{\top}|x-y|^{2 \gamma-2} .
$$

As $\left(u_{i}\right)_{i \in \mathcal{J}}$ (resp. $\left.\left(w^{i}\right)_{i \in \mathcal{J}}\right)$ is a subsolution (resp. supersolution) of (3.3) and taking into account (4.9), we obtain:

$$
-p_{u}^{n}-b\left(t_{n}, x_{n}\right)^{\top} \cdot q_{u}^{n}-\frac{1}{2} \operatorname{Tr}\left[\left(\sigma \sigma^{\top}\right)\left(t_{n}, x_{n}\right) M_{u}^{n}\right]-f_{j}\left(t_{n}, x_{n}, u_{j}\left(t_{n}, x_{n}\right), \sigma\left(t_{n}, x_{n}\right)^{\top} \cdot q_{u}^{n}\right) \leq 0
$$

and

$$
-p_{w}^{n}-b\left(t_{n}, y_{n}\right)^{\top} \cdot q_{w}^{n}-\frac{1}{2} \operatorname{Tr}\left[\left(\sigma \sigma^{\top}\right)\left(t_{n}, y_{n}\right) M_{w}^{n}\right]-f_{j}\left(t_{n}, y_{n}, w^{j}\left(t_{n}, y_{n}\right), \sigma\left(t_{n}, y_{n}\right)^{\top} \cdot q_{w}^{n}\right) \geq 0
$$

Making the difference between those two inequalities yields:

$$
\begin{array}{r}
-\left(p_{u}^{n}-p_{w}^{n}\right)-\left(b\left(t_{n}, x_{n}\right)^{\top} \cdot q_{u}^{n}-b\left(t_{n}, y_{n}\right)^{\top} \cdot q_{w}^{n}\right)-\frac{1}{2} \operatorname{Tr}\left[\left\{\sigma \sigma^{\top}\left(t_{n}, x_{n}\right) M_{u}^{n}-\sigma \sigma^{\top}\left(t_{n}, y_{n}\right) M_{w}^{n}\right\}\right] \\
-\left(f_{j}\left(t_{n}, x_{n}, u_{j}\left(t_{n}, x_{n}\right), \sigma^{\top}\left(t_{n}, x_{n}\right) \cdot q_{u}^{n}\right)-f_{j}\left(t_{n}, y_{n}, w^{j}\left(t_{n}, y_{n}\right), \sigma^{\top}\left(t_{n}, y_{n}\right) \cdot q_{w}^{n}\right)\right) \leq 0 .
\end{array}
$$

But

$$
\left.\left.\lim _{n \rightarrow \infty}\left\{\mid b\left(t_{n}, x_{n}\right)^{\top} \cdot q_{u}^{n}-b\left(t_{n}, y_{n}\right)^{\top} \cdot q_{w}^{n}\right)|+| \sigma\left(t_{n}, x_{n}\right)^{\top} \cdot q_{u}^{n}-\sigma\left(t_{n}, y_{n}\right)^{\top} \cdot q_{w}^{n}\right) \mid\right\}=0 .
$$

As usual taking into account (4.10) we have:

$$
\varlimsup_{n} \operatorname{Tr}\left[\left\{\sigma \sigma^{\top}\left(t_{n}, x_{n}\right) M_{u}^{n}-\sigma \sigma^{\top}\left(t_{n}, y_{n}\right) M_{w}^{n}\right\}\right] \leq 0
$$


Finally taking the limit in (4.11) and using the assumption (4.3) to obtain:

$$
-\lambda\left(u_{j}\left(t^{*}, x^{*}\right)-w^{j}\left(t^{*}, x^{*}\right)\right) \leq 0
$$

which is contradictory with (4.4) and then for any $j \in \mathcal{J}$ we have $u_{j} \leq w^{j}$.

Step 2: The general case.

Once more let $\left(u_{j}\right)_{j \in \mathcal{J}}$ (resp. $\left(w^{j}\right)_{j \in \mathcal{J}}$ ) be a subsolution (resp. supersolution) of (2.3). For $j \in \mathcal{J}$ let us set $\tilde{u}_{j}(t, x)=e^{\lambda t} u_{j}(t, x)$ and $\tilde{w}^{j}(t, x)=e^{\lambda t} w^{j}(t, x),(t, x) \in[0, T] \times \mathbb{R}^{k}$. Then $\left(\tilde{u}_{j}\right)_{j \in \mathcal{J}}\left(\operatorname{resp} .\left(\tilde{w}^{j}\right)_{j \in \mathcal{J}}\right)$ is a subsolution (resp. supersolution) of the following system of variational inequalities with oblique reflection: for any $i \in \mathcal{J}$,

$$
\left\{\begin{array}{l}
\min \left\{\tilde{v}_{i}(t, x)-\max _{j \in \mathcal{J}^{-i}}\left(-e^{\lambda t} g_{i j}(t, x)+\tilde{v}_{j}(t, x)\right),\right. \\
\left.\quad-\partial_{t} \tilde{v}_{i}(t, x)-\mathcal{L} \tilde{v}_{i}(t, x)+\lambda \tilde{v}_{i}(t, x)-e^{\lambda t} f_{i}\left(t, x, e^{-\lambda t} \tilde{v}^{i}(t, x), e^{-\lambda t} \sigma^{\top}(t, x) . D_{x} \tilde{v}^{i}(t, x)\right)\right\}=0 ; \\
\tilde{v}_{i}(T, x)=e^{\lambda T} h_{i}(x) .
\end{array}\right.
$$

Actually let $i \in \mathcal{J}$ and let $\varphi(t, x)$ be a $C^{1,2}$-function such that $\varphi-\tilde{u}_{i}$ has a minimum at $(t, x)$ and $\varphi(t, x)=\tilde{u}_{i}(t, x)$. Therefore $e^{-\lambda t} \varphi-u_{i}$ has a minimum at $(t, x)$ and $e^{-\lambda t} \varphi(t, x)=u_{i}(t, x)$. As $u_{i}$ is a subsolution then $\tilde{u}_{i}(T, x) \leq e^{\lambda T} h_{i}(x)$ and,

$$
\begin{aligned}
\min \left\{u_{i}(\right. & t, x)-\max _{j \in \mathcal{J}^{-i}}\left(-g_{i j}(t, x)+u_{j}(t, x)\right), \\
& \left.\quad-\partial_{t}\left(e^{-\lambda t} \varphi(t, x)\right)-\mathcal{L}\left(e^{-\lambda t} \varphi\right)(t, x)-f_{i}\left(t, x, e^{-\lambda t} \varphi(t, x), \sigma^{\top}(t, x) \cdot D_{x} e^{-\lambda t} \varphi(t, x)\right)\right\} \leq 0 .
\end{aligned}
$$

Now if

$$
u_{i}(t, x)-\max _{j \in \mathcal{J}^{-i}}\left(-g_{i j}(t, x)+u_{j}(t, x)\right) \leq 0
$$

then we have

$$
\tilde{u}_{i}(t, x)-\max _{j \in \mathcal{J}^{-i}}\left(-e^{\lambda t} g_{i j}(t, x)+\tilde{u}_{j}(t, x)\right),
$$

and the viscosity subsolution property is satisfied. If not, i.e., $u_{i}(t, x)-\max _{j \in \mathcal{J}^{-i}}\left(-g_{i j}(t, x)+u_{j}(t, x)\right)>0$ then:

$$
-\partial_{t}\left(e^{-\lambda t} \varphi(t, x)\right)-\mathcal{L}\left(e^{-\lambda t} \varphi\right)(t, x)-f_{i}\left(t, x, e^{-\lambda t} \varphi(t, x), \sigma^{\top}(t, x) . D_{x} e^{-\lambda t} \varphi(t, x)\right) \leq 0
$$

which implies

$$
-\partial_{t} \varphi(t, x)+\lambda \varphi(t, x)-\mathcal{L} \varphi(t, x)-e^{\lambda t} f_{i}\left(t, x, e^{-\lambda t} \tilde{u}_{i}(t, x), e^{-\lambda t} \sigma^{\top}(t, x) . D_{x} \varphi(t, x)\right) \leq 0
$$

Therefore once more the viscosity subsolution property is satisfied. As $i$ is arbitrary in $\mathcal{J}$, then $\left(\tilde{u}_{i}\right)_{i \in \mathcal{J}}$ is a viscosity subsolution for (4.15).

In the same way one can show that $\left(\tilde{w}^{j}\right)_{j \in \mathcal{J}}$ is a viscosity supersolution of (4.15), whence the claim. 
Next for $i \in \mathcal{J}$ let us set:

$$
F_{i}(t, x, u, z)=-\lambda u+e^{\lambda t} f_{i}\left(t, x, e^{-\lambda t} u, e^{-\lambda t} z\right) .
$$

Taking now $\lambda=\left(1+\max _{i=1, m} C^{i}\right)$, where $C^{i}$ is the Lipschitz constant of $f_{i}$ w.r.t. to $u$, to obtain that for $u \geq v, F_{i}(t, x, u, z)-F_{i}(t, x, v, z) \leq-(u-v)$. It means that $F_{i}$ satisfies the assumption (4.3). Therefore and according to the result proved in Step 1, for any $j \in \mathcal{J}$, we have $\tilde{u}_{j} \leq \tilde{w}^{j}$ and also $u_{j} \leq w^{j}$. The proof of the proposition is now complete.

Next, thanks to Proposition 4.1, we classically deduce both uniqueness and continuity results of any solution of (2.3) which belongs to $\Pi^{g}$. Actually if $\left(u_{i}\right)_{i \in \mathcal{J}}$ is a solution then $\left(u_{i}^{*}\right)_{i \in \mathcal{J}}\left(\operatorname{resp} .\left(u_{i *}\right)_{i \in \mathcal{J}}\right)$ is a subsolution (resp. supersolution) for the system (2.3) in the class $\Pi^{g}$, then we deduce that $u_{i}^{*} \leq u_{i *}$ and then $u_{i}^{*}=u_{i *}=u_{i}$, for any $i \in \mathcal{J}$. Whence the continuity of $\left(u_{i}\right)_{i \in \mathcal{J}}$. To sum up, we have:

Theorem 4 Assume that Assumptions (H2)-(i),(ii) and (H5) for $f_{i}, i \in \mathcal{J}$, are fulfilled. Then:

(i) The system of variational inequalities with inter-connected obstacles (2.3) has at most one solution in the class $\Pi^{g}$;

(ii) If the solution in $\Pi^{g}$ exists, it is necessarily continuous.

\section{Existence of a solution for the system}

\subsection{Case 1: $f_{i}$ depends only on $\left(y^{i}, z^{i}\right)$.}

In this specific case we have the following existence result:

Theorem 5 Under (H2), (H3), (H4) and (H5), the following system of variational inequalities with inter-connected obstacles

$$
\left\{\begin{array}{l}
\min \left\{v_{i}(t, x)-\max _{j \in \mathcal{J}^{-i}}\left(-g_{i j}(t, x)+v_{j}(t, x)\right)\right. \\
\left.\quad-\partial_{t} v_{i}(t, x)-\mathcal{L} v_{i}(t, x)-f_{i}\left(t, x, v_{i}(t, x), \sigma^{\top}(t, x) \cdot D_{x} v^{i}(t, x)\right)\right\}=0 \\
v_{i}(T, x)=h_{i}(x)
\end{array}\right.
$$

has a unique continuous solution $\left(v^{i}\right)_{i \in \mathcal{J}}$ in the class $\Pi^{g}$.

Proof: First note that the hypothesis (H2)-(iv) does not make any sense in consideration with (H5). Now let $\left(v^{i}\right)_{i \in \mathcal{J}}$ be the functions constructed in Prop. 3.1 which are associated with the solution of the system of reflected BSDEs with inter-connected obstacles associated with $\left(\left(f_{i}\right)_{i \in \mathcal{J}},\left(h_{i}\right)_{i \in \mathcal{J}},\left(g_{i j}\right)_{i, j \in \mathcal{J}}\right)$, which both exist under (H2)-(H5). The functions $v^{i}, i \in \mathcal{J}$, are of polynomial growth, thus locally bounded. Next let us show that they are viscosity solutions for the system (5.1). 
For any $i \in \mathcal{J}, v^{i}$ is $l s c$, then $v^{i}=v_{*}^{i}$. So let us show that the $m$-uplet $\left(v^{i}\right)_{i \in \mathcal{J}}$ is a viscosity supersolution to (5.1). First note that for any $i \in \mathcal{J}$,

$$
v^{i}=\lim _{n \rightarrow \infty} \nearrow v^{i, n}
$$

where $v^{i, n}$, for $n \geq 1$, is defined in (3.9) . By El-Karoui et al.'s result ([12], Thm. 8.5), $v^{i, n}$ is a viscosity solution of the following variational inequality or PDE with obstacle:

$$
\left\{\begin{aligned}
& \min \left\{v^{i, n}(t, x)\right.-\max _{j \in \mathcal{J}^{-i}}\left[v^{j, n-1}(t, x)-g_{i j}(t, x)\right] ; \\
&-\partial_{t} v^{i, n}-\mathcal{L} v^{i, n}(t, x)-f_{i}\left(t, x, v^{i, n}(t, x), \sigma(t, x)^{\top} D_{x} v^{i, n}(t, x)\right\}=0 ; \\
& v^{i, n}(T, x)=h_{i}(T, x) .
\end{aligned}\right.
$$

Now let us fix $i \in \mathcal{J}$, let $(t, x) \in\left[0, T\left[\times \mathbb{R}^{k}\right.\right.$ and $(p, q, M) \in \bar{J}^{-} v^{i}(t, x)$. By (5.2) and Lemma 6.1 in [8], there exist sequences

$$
n_{j} \rightarrow \infty, \quad\left(p_{j}, q_{j}, M_{j}\right) \in J^{-} v^{i, n_{j}}\left(t_{j}, x_{j}\right)
$$

such that:

$$
\left(t_{j}, x_{j}, v^{i, n_{j}}\left(t_{j}, x_{j}\right), p_{j}, q_{j}, M_{j}\right) \rightarrow\left(t, x, v^{i}(t, x), p, q, M\right) .
$$

Now from the viscosity supersolution property for $v^{i, n_{j}}$ we have:

$$
-p_{j}-b\left(t_{j}, x_{j}\right)^{\top} q_{j}-\frac{1}{2} \operatorname{Tr}\left(\sigma \sigma^{\top}\left(t_{j}, x_{j}\right) M_{j}\right)-f_{i}\left(t_{j}, x_{j}, v^{i, n_{j}}(t, x), \sigma\left(t_{j}, x_{j}\right)^{\top} q_{j}\right) \geq 0,
$$

and taking then the limit as $j \rightarrow \infty$ we obtain:

$$
-p-b(t, x)^{\top} q-\frac{1}{2} \operatorname{Tr}\left(\sigma \sigma^{\top}(t, x) M\right)-f_{i}\left(t, x, v^{i}(t, x), \sigma(t, x)^{\top} q\right) \geq 0 .
$$

As $v^{i}(t, x) \geq \max _{j \in \mathcal{J}^{-i}}\left(v^{j}(t, x)-g_{i j}(t, x)\right)$ and $v^{i}(T, x)=h_{i}(x)$ then $v^{i}$ is a viscosity supersolution for the following PDE with obstacle:

$$
\left\{\begin{array}{l}
\min \left\{v^{i}(t, x)-\max _{j \in \mathcal{J}^{-i}}\left[v^{j}(t, x)-g_{i j}(t, x)\right]\right. \\
\quad-\partial_{t} v^{i}-\mathcal{L} v^{i}(t, x)-f_{i}\left(t, x, v^{i}(t, x), \sigma(t, x)^{\top} D_{x} v^{i}(t, x)\right\}=0 \\
v^{i}(T, x)=h_{i}(x) .
\end{array}\right.
$$

Finally as $i$ is arbitrary in $\mathcal{J}$ then the $m$-uplet $\left(v^{1}, \ldots, v^{m}\right)$ is a viscosity supersolution for the system of variational inequalities (5.1).

Next let us show that $\left(v^{i *}\right)_{i \in \mathcal{J}}$ is a subsolution for (5.1). First let us show that for any $i \in \mathcal{J}$, $v^{i *}(T, x)=h_{i}(x)$. To begin with we are going to show that:

$$
\min \left\{v^{i *}(T, x)-h_{i}(x) ; v^{i *}(T, x)-\max _{j \in \mathcal{J}^{-i}}\left(v^{j *}(T, x)-g_{i j}(T, x)\right)\right\}=0
$$

Actually

$$
v^{i *}(T, x)=\varlimsup_{\left(t^{\prime}, x^{\prime}\right) \rightarrow(T, x), t^{\prime}<T} v^{i}\left(t^{\prime}, x^{\prime}\right) \geq \varlimsup_{\left(t^{\prime}, x^{\prime}\right) \rightarrow(T, x), t^{\prime}<T} v^{i, n}\left(t^{\prime}, x^{\prime}\right), \text { for any } n \geq 0
$$


therefore

$$
v^{i *}(T, x) \geq v^{i, n}(T, x)=h_{i}(x)
$$

since $v^{i, n}$ is continuous and at $t=T$ it equals to $h_{i}(x)$. On the other hand for any $(t, x)$ we have:

$$
\left.v^{i}(t, x) \geq \max _{j \in \mathcal{J}^{-i}}\left(v^{j}(t, x)-g_{i j}(t, x)\right)\right\}
$$

then

$$
v^{i *}(T, x) \geq \max _{j \in \mathcal{J}^{-i}}\left(v^{j *}(T, x)-g_{i j}(T, x)\right)
$$

which with (5.5) imply that:

$$
\min \left\{v^{i *}(T, x)-h_{i}(x) ; v^{i *}(T, x)-\max _{j \in \mathcal{J}^{-i}}\left(v^{j *}(T, x)-g_{i j}(T, x)\right)\right\} \geq 0 .
$$

Let us now show that the left-hand side of (5.6) cannot be positive. We first follow the same idea as in [3]. So let us suppose that for some $x_{0}$, there is $\varepsilon>0$ such that:

$$
\min \left\{v^{i *}\left(T, x_{0}\right)-h_{i}\left(x_{0}\right) ; v^{i *}\left(T, x_{0}\right)-\max _{j \in \mathcal{J}^{-i}}\left(v^{j *}\left(T, x_{0}\right)-g_{i j}\left(T, x_{0}\right)\right)\right\}=2 \varepsilon
$$

and let us construct a contradiction. Let $\left(t_{k}, x_{k}\right)_{k \geq 1}$ be a sequence in $[0, T] \times \mathbb{R}^{k}$ such that:

$$
\left(t_{k}, x_{k}\right) \rightarrow\left(T, x_{0}\right) \text { and } v^{i}\left(t_{k}, x_{k}\right) \rightarrow v^{i *}\left(T, x_{0}\right) \text { as } k \rightarrow \infty
$$

Since $v^{i, *}$ is usc and of polynomial growth and taking into account of (5.2), we can find a sequence $\left(\varrho^{n}\right)_{n \geq 0}$ of functions of $\mathcal{C}^{1,2}\left([0, T] \times \mathbb{R}^{k}\right)$ such that $\varrho^{n} \rightarrow v^{i, *}$ and, on some neighbourhood $B_{n}$ of $\left(T, x_{0}\right)$ we have:

$$
\min \left\{\varrho^{n}(t, x)-h_{i}(x), \varrho^{n}(t, x)-\max _{j \in \mathcal{J}^{-i}}\left(v^{j *}(t, x)-g_{i j}(t, x)\right)\right\} \geq \varepsilon, \forall(x, t) \in B_{n} .
$$

After possibly passing to a subsequence of $\left(t_{k}, x_{k}\right)_{k \geq 1}$ we can then assume that it holds on $B_{k}^{n}:=$ $\left[t_{k}, T\right] \times B\left(x_{k}, \delta_{n}^{k}\right)$ for some $\delta_{k}^{n} \in(0,1)$ small enough in such a way that $B_{k}^{n} \subset B$. Now since $v^{i, *}$ is locally bounded then there exists $\zeta>0$ such that $\left|v^{i, *}\right| \leq \zeta$ on $B_{n}$. We can then assume that $\varrho^{n} \geq-2 \zeta$ on $B_{n}$. Next let us define:

$$
\varrho_{k}^{n}(t, x):=\varrho^{n}(t, x)+\frac{4 \zeta\left|x-x_{k}\right|^{2}}{\left(\delta_{k}^{n}\right)^{2}}+\sqrt{T-t}
$$

Note that $\tilde{\varrho}_{k}^{n} \geq \varrho^{n}$ and

$$
\left(v^{i, *}-\tilde{\varrho}_{k}^{n}\right)(t, x) \leq-\zeta \text { for }(t, x) \in\left[t_{k}, T\right] \times \partial B\left(x_{k}, \delta_{k}^{n}\right)
$$

Next since $\partial_{t}(\sqrt{T-t}) \rightarrow-\infty$ as $t \rightarrow T$, we can choose $t_{k}$ large enough in front of $\delta_{k}^{n}$ and the derivatives of $\varrho^{n}$ to ensure that

$$
-\mathcal{L} \tilde{\varrho}_{k}^{n}(t, x) \geq 0 \text { on } B_{n}^{k}
$$


Next let us consider the following stopping time $\theta_{n}^{k}:=\inf \left\{s \geq t_{k},\left(s, X_{s}^{t_{k}, x_{k}}\right) \in B_{n}^{k^{c}}\right\} \wedge T$ where $B_{n}^{k^{c}}$ is the complement of $B_{n}^{k}$, and $\vartheta_{k}:=\inf \left\{s \geq t_{k}, v^{i}\left(s, X_{s}^{t_{k}, x_{k}}\right)=\max _{j \in \mathcal{J}^{-i}}\left(v^{j}\left(s, X_{s}^{t_{k}, x_{k}}\right)-g_{i j}\left(s, X_{s}^{t_{k}, x_{k}}\right)\right)\right\} \wedge T$. Applying now Itô's formula to the process $\left(\tilde{\varrho}_{k}^{n}\left(s, X_{s}\right)\right)$ stopped at time $\theta_{n}^{k} \wedge \vartheta_{k}$ and taking into account (5.7), (5.8) and (5.9) to obtain:

$$
\begin{aligned}
\tilde{\varrho}_{k}^{n}\left(t_{k}, x_{k}\right)= & \mathbb{E}\left[\tilde{\varrho}_{k}^{n}\left(\theta_{n}^{k} \wedge \vartheta_{k}, X_{\theta_{n}^{k} \wedge \vartheta_{k}}^{t_{k}, x_{k}}\right)-\int_{t_{k}}^{\theta_{n}^{k} \wedge \vartheta_{k}} \mathcal{L} \tilde{\varrho}_{k}^{n}\left(r, X_{r}^{t_{k}, x_{k}}\right) d r\right] \\
\geq & \mathbb{E}\left[\varrho_{k}^{n}\left(\theta_{n}^{k}, X_{\theta_{n}^{k}}^{t_{k}, x_{k}}\right) \mathbb{1}_{\left[\theta_{n}^{k} \leq \vartheta_{k}\right]}+\tilde{\varrho}_{k}^{n}\left(\vartheta_{k}, X_{\vartheta_{k}}^{t_{k}, x_{k}}\right) \mathbb{1}_{\left[\vartheta_{k}<\theta_{n}^{k}\right]}\right] \\
= & \mathbb{E}\left[\left\{\tilde{\varrho}_{k}^{n}\left(\theta_{n}^{k}, X_{\theta_{n}^{k}}^{t_{k}, x_{k}}\right) \mathbb{1}_{\left[\theta_{n}^{k}<T\right]}+\tilde{\varrho}_{k}^{n}\left(T, X_{T}^{t_{k}, x_{k}}\right) \mathbb{1}_{\left[\theta_{n}^{k}=T\right]}\right\} \mathbb{1}_{\left[\theta_{n}^{k} \leq \vartheta_{k}\right]}+\tilde{\varrho}_{k}^{n}\left(\vartheta_{k}, X_{\vartheta_{k}}^{t_{k}, x_{k}}\right) \mathbb{1}_{\left[\vartheta_{k}<\theta_{n}^{k}\right]}\right] \\
\geq & \mathbb{E}\left[\left\{\left(v^{i *}\left(\theta_{n}^{k}, X_{\theta_{n}^{k}}^{t_{k}, x_{k}}\right)+\zeta\right) \mathbb{1}_{\left[\theta_{n}^{k}<T\right]}+\left(\varepsilon+h_{i}\left(T, X_{T}^{t_{k}, x_{k}}\right)\right) \mathbb{1}_{\left[\theta_{n}^{k}=T\right]}\right\} \mathbb{1}_{\left[\theta_{n}^{k} \leq \vartheta_{k}\right]}\right. \\
& \left.\quad \quad \quad\left\{\varepsilon+\max _{j \in \mathcal{J}^{-i}}\left(v^{j *}\left(\vartheta_{k}, X_{\vartheta_{k}}^{t_{k}, x_{k}}\right)-g_{i j}\left(\vartheta_{k}, X_{\vartheta_{k}}^{t_{k}, x_{k}}\right)\right)\right\} \mathbb{1}_{\left[\vartheta_{k}<\theta_{n}^{k}\right]}\right] \\
\geq & \mathbb{E}\left[\left\{\left(v^{i}\left(\theta_{n}^{k}, X_{\theta_{n}^{k}}^{t_{k}, x_{k}}\right)+\zeta\right) \mathbb{1}_{\left[\theta_{n}^{k}<T\right]}+\left(\varepsilon+h_{i}\left(T, X_{T}^{t_{k}, x_{k}}\right)\right) \mathbb{1}_{\left[\theta_{n}^{k}=T\right]}\right\} \mathbb{1}_{\left[\theta_{n}^{k} \leq \vartheta_{k}\right]}\right. \\
& \left.\quad+\left\{\varepsilon+\max _{j \in \mathcal{J}}\left(v^{j}\left(\vartheta_{k}, X_{\vartheta_{k}}^{t_{k}, x_{k}}\right)-g_{i j}\left(\vartheta_{k}, X_{\vartheta_{k}}^{t_{k}, x_{k}}\right)\right)\right\} \mathbb{1}_{\left[\vartheta_{k}<\theta_{n}^{k}\right]}\right] \text { since } v^{i *} \geq v^{i} \\
\geq & \mathbb{E}\left[v^{i}\left(\theta_{n}^{k} \wedge \vartheta_{k}, X_{\theta_{n}^{k} \wedge \vartheta_{k}}^{t_{k}, x_{k}}\right)\right]+\zeta \wedge \varepsilon \\
= & \mathbb{E}\left[v^{i}\left(t_{k}, x_{k}\right)-\int_{t_{k}}^{\theta_{n}^{k} \wedge \vartheta_{k}} f_{i}\left(s, X_{s}^{t_{k}, x_{k}}, v^{i}\left(s, X_{s}^{t_{k}, x_{k}}\right), Z_{s}^{i, t_{k}, x_{k}}\right) d s\right]+\zeta \wedge \varepsilon
\end{aligned}
$$

since on $\left[t_{k}, \vartheta_{k}\right], d K^{i, t, x}=0$. Finally since $v^{i}$ and $\left\|Z^{i}\right\|_{\mathcal{H}^{2, d}}(t, x)$ belong to $\Pi^{g}$ (see Prop 3.1 and Remark 2) and taking into account (3.2) and assumption (H2)-(iii), we easily deduce that

$$
\lim _{k \rightarrow \infty} \mathbb{E}\left[\int_{t_{k}}^{\theta_{n}^{k} \wedge \vartheta_{k}} f_{i}\left(s, X_{s}^{t_{k}, x_{k}}, v^{i}\left(s, X_{s}^{t_{k}, x_{k}}\right), Z_{s}^{i, t_{k}, x_{k}}\right) d s\right]=0 .
$$

Therefore taking the limit in the previous inequalities yields:

$$
\lim _{k \rightarrow \infty} \tilde{\varrho}_{k}^{n}\left(t_{k}, x_{k}\right)=\lim _{k \rightarrow \infty} \varrho^{n}\left(t_{k}, x_{k}\right)+\sqrt{T-t_{k}}=\varrho^{n}\left(T, x_{0}\right) \geq \lim _{k \rightarrow \infty} v^{i}\left(t_{k}, x_{k}\right)+\zeta \wedge \varepsilon=v^{* i}\left(T, x_{0}\right)+\zeta \wedge \varepsilon .
$$

But this is a contradiction since $\varrho^{n} \rightarrow v^{i *}$ pointwisely as $n \rightarrow \infty$. Thus for any $(t, x) \in[0, T] \times \mathbb{R}^{k}$ we have:

$$
\min \left\{v^{i *}(T, x)-h_{i}(x) ; v^{i *}(T, x)-\max _{j \in \mathcal{J}^{-i}}\left(v^{j *}(T, x)-g_{i j}(T, x)\right)\right\}=0 .
$$

Let us now show that $v^{i *}(T, x)=h_{i}(x)$. So suppose that $v^{i *}(T, x)>h_{i}(x)$, then for for some $j \in \mathcal{J}^{-i}$ we have

$$
v^{i *}(T, x)=v^{j *}(T, x)-g_{i j}(T, x) .
$$

But once more we have $v^{j *}(T, x)>h_{j}(T, x)$. Otherwise, i.e. if $v^{j *}(T, x)=h_{j}(T, x)$, we would have:

$$
h_{i}(x)<h_{j}(x)-g_{i j}(T, x)
$$

which is contradictory with (H4). Therefore there exists $\ell \in \mathcal{J}^{-j}$ such that:

$$
v^{j *}(T, x)=v^{\ell *}(T, x)-g_{j \ell}(T, x) \text { and then } v^{i *}(T, x)=v^{\ell *}(T, x)-g_{j \ell}(T, x)-g_{i j}(T, x) .
$$

Repeating this reasoning as many times as necessary we obtain a sequence of different indices $i_{1}, \ldots, i_{l}$ such that

$$
v^{i_{1} *}(T, x)=v^{i_{1} *}(T, x)-\left(g_{i_{1} i_{2}}(T, x)+\cdots+g_{i_{l-1} i_{l}}(T, x)+g_{i_{l} i_{1}}(T, x)\right)
$$


which is contradictory with (H3)-(ii). Thus for any $i \in \mathcal{J}$ we have:

$$
\forall x \in \mathbb{R}^{k}, v^{i *}(T, x)=h_{i}(x) .
$$

Let us now show that $\left(v^{i *}\right)_{i \in \mathcal{J}}$ is a subsolution to (5.1). First note that since $v^{i, n} \nearrow v^{i}$ and $v^{i, n}$ is continuous then we have (see e.g. [26], pp.91)

$$
v^{i *}(t, x)=\lim _{n \rightarrow \infty} \sup ^{*} v^{i, n}(t, x)=\lim \sup _{n \rightarrow \infty, t^{\prime} \rightarrow t, x^{\prime} \rightarrow x} v^{i, n}\left(t^{\prime}, x^{\prime}\right) .
$$

Next let us fix $i \in \mathcal{J}$ and let $(t, x) \in\left[0, T\left[\times \mathbb{R}^{k}\right.\right.$ be such that

$$
v^{i *}(t, x)-\max _{\ell \in \mathcal{J}^{-i}}\left(v^{\ell *}(t, x)-g_{i \ell}(t, x)\right)>0 .
$$

Let $(p, q, M) \in \bar{J}^{+} v^{i *}(t, x)$. By (5.10) and Lemma 6.1 in [8], there exist sequences

$$
n_{j} \rightarrow \infty, \quad\left(p_{j}, q_{j}, M_{j}\right) \in J^{+} v^{i, n_{j}}\left(t_{j}, x_{j}\right)
$$

such that:

$$
\lim _{j \rightarrow \infty}\left(t_{j}, x_{j}, v^{i, n_{j}}\left(t_{j}, x_{j}\right), p_{j}, q_{j}, M_{j}\right)=\left(t, x, v^{i *}(t, x), p, q, M\right) .
$$

Now from the viscosity subsolution property for $v^{i, n_{j}}$ at $\left(t_{j}, x_{j}\right)$ (see $\lcm{5.3}$ ), for any $j \geq 0$, we have:

$$
\begin{aligned}
\min \left\{v^{i, n_{j}}\right. & \left(t_{j}, x_{j}\right)-\max _{\ell \in \mathcal{J}^{-i}}\left(v^{\ell, n_{j}-1}\left(t_{j}, x_{j}\right)-g_{i \ell}\left(t_{j}, x_{j}\right)\right) ; \\
& \left.\quad-p_{j}-b\left(t_{j}, x_{j}\right)^{\top} q_{j}-\frac{1}{2} \operatorname{Tr}\left(\sigma \sigma^{\top}\left(t_{j}, x_{j}\right) M_{j}\right)-f_{i}\left(t_{j}, x_{j}, v^{i, n_{j}}\left(t_{j}, x_{j}\right), \sigma\left(t_{j}, x_{j}\right)^{\top} q_{j}\right)\right\} \leq 0 .
\end{aligned}
$$

Next the definition of $v^{\ell *}$ implies that

$$
v^{\ell *}(t, x) \geq \lim \sup _{j \rightarrow \infty} v^{\ell, n_{j}}\left(t_{j}, x_{j}\right),
$$

therefore by (5.11), there exists $j_{0} \geq 0$, such that if $j \geq j_{0}$ we have

$$
v^{i, n_{j}}\left(t_{j}, x_{j}\right)>\max _{\ell \in \mathcal{J}^{-i}}\left(v^{\ell, n_{j}}\left(t_{j}, x_{j}\right)-g_{i j}\left(t_{j}, x_{j}\right)\right) .
$$

Then (5.12) implies that, for any $j \geq j_{0}$,

$$
-p_{j}-b\left(t_{j}, x_{j}\right)^{\top} q_{j}-\frac{1}{2} \operatorname{Tr}\left(\sigma \sigma^{\top}\left(t_{j}, x_{j}\right) M_{j}\right)-f_{i}\left(t_{j}, x_{j}, v^{i, n_{j}}\left(t_{j}, x_{j}\right), \sigma\left(t_{j}, x_{j}\right)^{\top} q_{j}\right) \leq 0 .
$$

Taking the limit as $j \rightarrow \infty$ we deduce that

$$
-p-b(t, x)^{\top} q-\frac{1}{2} \operatorname{Tr}\left(\sigma \sigma^{\top}(t, x) M\right)-f_{i}\left(t, x, v^{i *}(t, x), \sigma(t, x)^{\top} q\right) \leq 0
$$

since $f_{i}$ is uniformly continuous in $(t, x)$ and Lipschitz in $\left(y^{i}, z\right)$. Then

$$
\begin{aligned}
& \min \left\{v^{i *}(t, x)-\max _{\ell \in \mathcal{J}^{-i}}\left(v^{\ell *}(t, x)-g_{i \ell}(t, x)\right) ;\right. \\
& \left.\quad-p-b(t, x)^{\top} q-\frac{1}{2} \operatorname{Tr}\left(\sigma \sigma^{\top}(t, x) M\right)-f_{i}\left(t, x, v^{i *}(t, x), \sigma(t, x)^{\top} q\right)\right\} \leq 0
\end{aligned}
$$

which means that $v^{i *}$ is a viscosity subsolution for (5.1). Thus the $m$-uplet $\left(v^{i}\right)_{i \in \mathcal{J}}$ is a solution for (5.1) and Theorem 4 implies that it is continuous and unique.

As a by-product we obtain: 
Corollary 1 Under (H1)-(H5), there exist deterministic continuous functions $\left(v^{i}(t, x)\right)_{i \in \mathcal{J}}$ which belong to $\Pi^{g}$ unique solution of (4.15) and such that the unique solution of the system of reflected BSDEs with inter-connected obstacles associated with $\left(\left(f_{i}\right)_{i \in \mathcal{J}},\left(h_{i}\right)_{i \in \mathcal{J}},\left(g_{i j}\right)_{i, j \in \mathcal{J}}\right)$ has the following representation:

$$
\forall i \in \mathcal{J}, \forall(t, x) \in[0, T] \times \mathbb{R}^{k}, \forall s \in[t, T], \quad Y_{s}^{i ; t, x}=v^{i}\left(s, X_{s}^{t, x}\right)
$$

\subsection{Case 2: Existence and uniqueness in the general setting}

In this section we deal with the issue of existence and uniqueness of the solution of (2.3) in its general form, i.e., when the functions $f_{i}, i \in \mathcal{J}$, depend not only on $y^{i}$ but also on the other components $\left(y_{1}, \ldots, y_{m}\right)$. More precisely, we successively consider the cases when either $f_{i}$ or $-f_{i}$ satisfies (H2)-(iv) for any $i \in \mathcal{J}$. Considering the first case, we have:

Theorem 6 Under (H2), (H3), (H4), the system of variational inequalities with inter-connected obstacles (2.3) has a continuous solution $\left(v^{1}, \ldots, v^{m}\right)$ in the class $\Pi^{g}$.

Proof: We first prove existence. Let $\lambda \in \mathbb{R}$ and for $i \in \mathcal{J}$, let $F_{i}$ be the function defined by:

$$
F_{i}\left(t, x, y^{1}, \ldots, y^{m}, z\right)=e^{\lambda t} f_{i}\left(t, x, e^{-\lambda t} y_{1}, \ldots, e^{-\lambda t} y_{m}, e^{-\lambda t} z_{i}\right)-\lambda y_{i}
$$

Since $f_{i}$ is uniformly Lipschitz w.r.t. $y_{i}$ then $F_{i}$ is so and for $\lambda$ small enough $(\lambda<0)$ the function $F_{i}$ is non-decreasing in all variables $\left(y_{1}, \ldots, y_{m}\right)$. Next thanks to Theorems 1 and 2 , there exist processes $\left(Y^{i}, Z^{i}, K^{i}\right)_{i \in \mathcal{J}}$ solution of the system of reflected BSDEs with interconnected obstacles associated with $\left(\left(F_{i}\left(t, x, y^{1}, \ldots, y^{m}, z\right)\right)_{i \in \mathcal{J}},\left(e^{\lambda T} h_{i}\left(X_{T}^{t, x}\right)\right)_{i \in \mathcal{J}},\left(e^{\lambda t} g_{i j}\right)_{i, j \in \mathcal{J}}\right)$. Additionally thanks to Proposition 3.1, there exist deterministic $l s c$ functions $v^{i}, i \in \mathcal{J}$, such that:

$$
\forall i \in \mathcal{J}, \forall t \leq T, \forall s \in[t, T], Y_{s}^{i}=v^{i}\left(s, X_{s}^{t, x}\right)
$$

Let us now analyze the decreasing scheme. First let us consider $(\bar{Y}, \bar{Z})$ the solution of the following standard BSDE:

$$
\left\{\begin{array}{l}
\bar{Y} \in \mathcal{S}^{2}, \bar{Z} \in \mathcal{H}^{2, d} \\
\bar{Y}_{s}=\max _{i=1, m} e^{\lambda T} h_{i}\left(X_{T}^{t, x}\right)+\int_{s}^{\top}\left[\max _{i=1, m} F_{i}\right]\left(r, X_{r}^{t, x}, \bar{Y}_{r}, \ldots, \bar{Y}_{r}, \bar{Z}_{r}\right) d r-\int_{s}^{\top} \bar{Z}_{r} d B_{r} s \leq T .
\end{array}\right.
$$

Next for any $i \in \mathcal{J}$, let us set $Y^{i, 0}=\bar{Y}$ and for $n \geq 1$ let us define $\left(Y^{i, n}, Z^{i, n}, K^{i, n}\right)$ by:

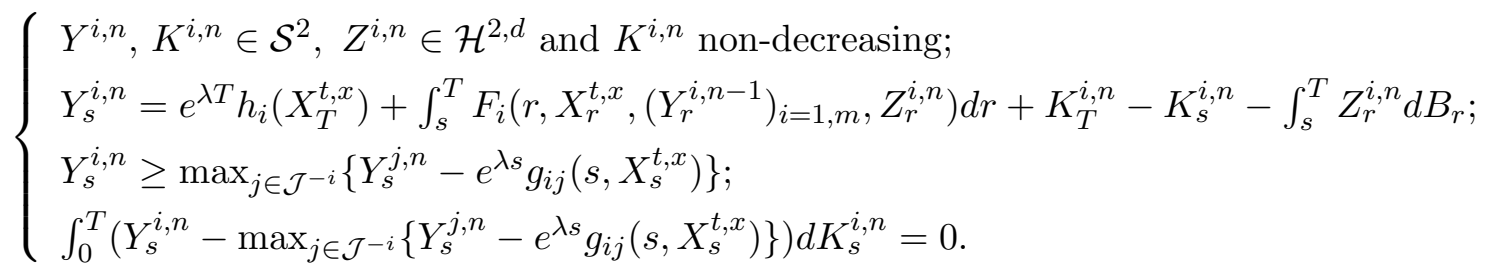


First note that the existence of $\bar{Y}$ is obvious by Pardoux-Peng's result [24] and then we easily deduce by induction:

(i) for any $n \geq 1$, there exists a unique $m$-uplet of processes $\left(Y^{i, n}, Z^{i, n}, K^{i, n}\right), i \in \mathcal{J}$, ;

(ii) for any $n \geq 1$, there exist deterministic continuous functions $v^{i, n}, i \in \mathcal{J}$, such that:

$$
\forall i \in \mathcal{J}, \forall t \leq T, \forall s \in[t, T], Y_{s}^{i, n}=v^{i, n}\left(s, X_{s}^{t, x}\right)
$$

(iii) for any $i \in \mathcal{J}$ and $n \geq 0, Y^{i, n+1} \leq Y^{i, n}$ and $v^{i, n} \geq v^{i, n+1}$;

(iv) for any $i \in \mathcal{J}$, the sequence $\left(Y^{i, n}\right)_{n}$ converges to $Y^{i}$ in $\mathcal{H}^{2,1}$, where such a process $Y^{i}$ has been introduced in equation (5.15).

Actually for (i), we just need to use Theorem 1 and 2 since $Y^{i, 0}$ exists. To show (ii), we use induction: obviously the property is valid for $n=0$ (see e.g. [13], Thm.4.1). Next if the property holds for some $n$ then it holds also for $n+1$ in using Corollary 1. As for (iii), the property is true for $n=0$ since $\left(\left(\bar{Y}^{i}, \bar{Z}^{i}, \bar{K}^{i}\right)=(\bar{Y}, \bar{Z}, 0)\right)_{i \in \mathcal{J}}$ is the unique solution of the system associated with

$\left.\left(\bar{f}_{i}:=\left[\max _{i=1, m} F_{i}\right]\left(r, X_{r}^{t, x}, y^{1}, \ldots, y^{m}, z\right)\right)_{i \in \mathcal{J}},\left(\bar{h}_{i}:=\max _{i=1, m} e^{\lambda T} h_{i}\left(X_{T}^{t, x}\right)\right)_{i \in \mathcal{J}},\left(e^{\lambda t} g_{i j}\right)_{i, j \in \mathcal{J}}\right)$ and then it is just enough to use the comparison result of Remark 1. Next if the property is valid for some $n$ then it is also valid for $n+1$, in using once more comparison since $F_{i}\left(t, x,\left(y^{i}\right)_{i \in \mathcal{J}}, z\right), i \in \mathcal{J}$, is non-decreasing in $\left(y^{i}\right)_{i \in \mathcal{J}}$. Finally (iv) holds true because the mapping $\Phi$ defined in (3.16) is a contraction in $\mathcal{H}^{2, m}$ and we obviously have:

$$
\forall n \geq 0,\left(Y^{i, n+1}\right)_{i=1, m}=\Phi\left(\left(Y^{i, n}\right)_{i=1, m}\right) .
$$

Let us now show that the deterministic functions $\left(v^{i}\right)_{i \in \mathcal{J}}$ of (5.15) are continuous. Recall that thanks to Proposition 3.1 each $v^{i}$ is the limit of some increasing sequence and hence it is $l s c$ : therefore, it is enough to show that they are upper semicontinuous. But from the estimate given in (3.17), we know that:

$$
\forall(t, x) \in[0, T] \times \mathbb{R}^{k},\left|v^{i}(t, x)-v^{i, n}(t, x)\right|=\mathbb{E}\left[\left|Y_{t}^{i}-Y_{t}^{i, n}\right|\right] \leq C\left\|\left(Y^{i}\right)_{i \in \mathcal{J}}-\left(Y^{i, n-1}\right)_{i \in \mathcal{J}}\right\|_{\mathcal{H}^{2, m}} .
$$

As $\lim _{n \rightarrow \infty}\left\|\left(Y^{i}\right)_{i \in \mathcal{J}}-\left(Y^{i, n-1}\right)_{i \in \mathcal{J}}\right\|_{\mathcal{H}^{2, m}}=0$ then for any $i \in \mathcal{J}$, the sequence $\left(v^{i, n}\right)_{n}$ defined in (5.17) converges pointwisely and decreasingly (from (5.17)-(iii)) to $v^{i}$. As a decreasing limit of continuous functions, $v^{i}$ is $u s c$ and then continuous. It follows that the solution $\left(y^{i}, z^{i}, k^{i}\right)_{i \in \mathcal{J}}$ of the system of reflected BSDEs with oblique associated with $\left(\left(f_{i}\left(s, X_{s}^{t, x}, y^{1}, \ldots, y^{m}, z\right)\right)_{i \in \mathcal{J}},\left(h_{i}\left(X_{T}^{t, x}\right)\right)_{i \in \mathcal{J}},\left(g_{i j}\left(s, X_{s}^{t, x}\right)\right)_{i, j \in \mathcal{J}}\right)$ has the following representation:

$$
\forall i \in \mathcal{J}, \forall t \leq T, \forall s \in[t, T], \forall x \in \mathbb{R}^{k}, y_{s}^{i}=e^{-\lambda s} v^{i}\left(s, X_{s}^{t, x}\right)
$$

As $v^{i}, i \in \mathcal{J}$, is continuous and of polynomial growth then using the result by El-Karoui et al.([12], Thm. 8.5) related to connection between solutions of reflected BSDEs and viscosity solutions of PDE with obstacles we deduce that $\left(e^{-\lambda t} v^{i}(t, x)\right)_{i \in \mathcal{J}}$ is a viscosity solution for system (2.3). 
As previously mentioned, we now consider the case when the functions $-f_{i}, i \in \mathcal{J}$, verify (H2)-(iv). Then we have:

Theorem 7 Assume that assumptions (H3), (H4) are fulfilled and that the functions $\left(-f_{i}\right)_{i \in \mathcal{J}}$ verify (H2). Then the system of variational inequalities with inter-connected obstacles (2.3) has a continuous solution $\left(v^{1}, \ldots, v^{m}\right)$ in the class $\Pi^{g}$.

Proof: We first prove existence of a candidate to be a viscosity solution of the system. As previously, we will relate it to the unique solution of the multidimensional reflected BSDE (3.3).

\section{Step 1: Construction}

For $i \in \mathcal{J}$, let $F_{i}$ be defined as in (5.14). Choosing $\lambda$ large enough, we obtain that each $F^{i}$ is decreasing with respect to all the variables $y_{j}, j=1, \cdots, m$. Next let us consider the following iterative Picard scheme: for any $i \in \mathcal{J}, Y^{i, 0}=0$ and for $n \geq 1$, we define $\left(Y^{i, n}\right)_{i \in \mathcal{J}}$ by:

$$
\left(Y^{1, n}, \ldots, Y^{m, n}\right)=\Phi\left(\left(Y^{1, n-1}, \ldots, Y^{m, n-1}\right)\right) .
$$

Therefore we know from Theorem 2 that the sequence $\left(Y^{i, n}\right)_{i \in \mathcal{J}}$ converges in $\mathcal{H}^{2, m}$ to the unique solution $\left(Y^{i}\right)_{i \in \mathcal{J}}$ of the system of reflected BSDEs with oblique reflection associated with $\left(\left(F_{i}\left(s, X_{s}^{t, x}, y^{1}, \ldots, y^{m}, z\right)\right)_{i \in \mathcal{J}},\left(e^{\lambda T} h_{i}\left(X_{T}^{t, x}\right)\right)_{i \in \mathcal{J}},\left(e^{\lambda t} g_{i j}\left(s, X_{s}^{t, x}\right)\right)_{i, j \in \mathcal{J}}\right)$.

Next using Proposition 3.1 and an induction argument, we deduce the existence of continuous functions with polynomial growth such that:

$$
\forall n \geq 0, \forall i \in \mathcal{J}, \forall(t, x) \in[0, T] \times \mathbb{R}^{k}, \forall s \in[t, T], Y_{s}^{i, n}=v^{i, n}\left(s, X_{s}^{t, x}\right) .
$$

But from Theorem 3-(a) we have: for any $i, n, m$ and $s \leq T$,

$$
\begin{aligned}
& \mathbb{E}\left[\left|Y_{s}^{i, n}-Y_{s}^{i, m}\right|^{2}\right] \leq C\left\|\left(Y^{i, n-1}\right)_{i \in \mathcal{J}}-\left(Y^{i, m-1}\right)_{i \in \mathcal{J}}\right\|_{\mathcal{H}^{2, m}}^{2} \text { and } \\
& \mathbb{E}\left[\left|Y_{s}^{i}-Y_{s}^{i, m}\right|^{2}\right] \leq C\left\|\left(Y^{i}\right)_{i \in \mathcal{J}}-\left(Y^{i, m-1}\right)_{i \in \mathcal{J}}\right\|_{\mathcal{H}^{2, m}}^{2},
\end{aligned}
$$

where, to obtain the last inequality, we rely on the characterization of the solution $\left(Y^{i}\right)_{i \in \mathcal{J}}$ constructed in [7], i.e., $\left(Y^{i}\right)_{i \in \mathcal{J}}=\Phi\left(\left(Y^{i}\right)_{i \in \mathcal{J}}\right)$. Taking $s=t$ we obtain: $\forall(t, x) \in[0, T] \times \mathbb{R}^{k}$,

$$
\mathbb{E}\left[\left|Y_{t}^{i, n}-Y_{t}^{i, m}\right|\right]=\left|v^{i, n}(t, x)-v^{i, m}(t, x)\right| \leq C\left\|\left(Y^{i, n-1}\right)_{i \in \mathcal{J}}-\left(Y^{i, m-1}\right)_{i \in \mathcal{J}}\right\|_{\mathcal{H}^{2, m}} .
$$

As the sequence $\left(\left(Y^{i, n}\right)_{i \in \mathcal{J}}\right)_{n \geq 0}$ is convergent in $\mathcal{H}^{2, m}$ then it is of Cauchy type which implies that $\left(v^{i, n}\right)_{n \geq 0}$ is so and then converges pointwisely to a deterministic function $v^{i}$, for any $i \in \mathcal{J}$. Thus going back to (5.19) we deduce that:

$$
\forall i \in \mathcal{J}, \forall s \in[t, T], \mathbb{P}-\text { a.s., } Y_{s}^{i}=v^{i}\left(s, X_{s}^{t, x}\right) .
$$

Let us now show that $v^{i}, i \in \mathcal{J}$, belongs to $\Pi^{g}$. Actually since $\Phi$ is a contraction in $\left(\mathcal{H}^{2, m},\|\cdot\|_{\beta_{0}}\right)$ and by some induction procedure on $n$ we get:

$$
\forall n, q \geq 0,\left\|\left(Y^{i, n+q}\right)_{i \in \mathcal{J}}-\left(Y^{i, n}\right)_{i \in \mathcal{J}}\right\|_{\beta_{0}} \leq \frac{C_{\Phi}^{n}}{1-C_{\Phi}}\left\|\left(Y^{i, 1}\right)_{i \in \mathcal{J}}\right\|_{\beta_{0}} ;
$$


with $C_{\Phi}$ (such that $0<C_{\Phi}<1$ ) which is the contraction constant of the mapping $\Phi$ (constant which is independent of $(t, x))$. As the norms $\|$.$\| and \|\cdot\|_{\beta_{0}}$ are equivalent then there exists a constant $C_{1}$ such that:

$$
\forall n, q \geq 0,\left\|\left(Y^{i, n+q}\right)_{i \in \mathcal{J}}-\left(Y^{i, n}\right)_{i \in \mathcal{J}}\right\| \leq C_{1} C_{\Phi}^{n}\left\|\left(Y^{i, 1}\right)_{i \in \mathcal{J}}\right\|
$$

Taking now the limit as $q$ goes to $+\infty$ and in view of (5.19) and (5.20), if we then take $s=t$ we deduce that:

$$
\forall(t, x) \in[0, T] \times \mathbb{R}^{k},\left|v^{i}(t, x)-v^{i, n}(t, x)\right| \leq C_{2}\left\|\left(Y^{i, 1}\right)_{i \in \mathcal{J}}\right\|
$$

Finally one can check easily that $\left\|\left(Y^{i, 1}\right)_{i \in \mathcal{J}}\right\|(t, x)$ is of polynomial growth (since $\mathbb{E}\left[\sup _{s \leq T}\left|X_{s}^{t, x}\right|^{\gamma}\right]$ belongs to $\Pi^{g}$ for any $\gamma \geq 0$, see (3.2) ) and since $v^{i, n}$ is so, then we deduce that $v^{i}$ is also of polynomial growth for any $i \in \mathcal{J}$.

Step 2: Continuity of $v^{i}, i \in \mathcal{J}$.

We again rely on the convergence result of any sequence $\left(Y^{i, n}\right)_{n}$ constructed via the Picard iterative scheme. So let us initialize the scheme as follows:

$$
\forall i \in\{1, \cdots, m\}, \forall s \leq T, \quad Y_{s}^{i, 0}=C\left(1+\left|X_{s}^{t, x}\right|^{p}\right)
$$

with the constant $C$ and the integer $p$ given by the fact that $v^{i}$ is in $\Pi^{g}$.

Next let $\left(Y^{i, t, x}\right)_{i=\{1, \cdots, m\}}$ be the unique solution of the multidimensional RBSDE associated with $\left(\left(F_{i}\left(s, X_{s}^{t, x}, y^{1}, \ldots, y^{m}, z\right)\right)_{i \in \mathcal{J}},\left(e^{\lambda T} h_{i}\left(X_{T}^{t, x}\right)\right)_{i \in \mathcal{J}},\left(e^{\lambda t} g_{i j}\left(s, X_{s}^{t, x}\right)\right)_{i, j \in \mathcal{J}}\right)$ then we have $Y_{s}^{i, t, x}=v^{i}\left(s, X_{s}^{t, x}\right)$ for any $s \in[t, T]$ and $i \in \mathcal{J}$. By definition of $Y^{i, 0}$ in 5.21 and the hypothesis on $v^{i}$, we obtain

$$
\mathbb{P}-\text { a.s. }, \forall i \in \mathcal{J}, \forall s \in[t, T], Y_{s}^{i, t, x} \leq Y_{s}^{i, 0}
$$

Let us now prove by induction that for any $n \geq 0$ we have:

$$
\forall n \in \mathbb{N}, \quad \forall i \in \mathcal{J}, \forall s \in[t, T], Y_{s}^{i, 2 n+1} \leq Y_{s}^{i} \leq Y_{s}^{i, 2 n}
$$

For $n=0$, the inequality of the right-hand side is already true. Let consider the left-hand side. Recalling that by construction we have $\left(Y^{i, 1}\right)_{i \in \mathcal{J}}=\Phi\left(\left(Y^{i, 0}\right)_{i \in \mathcal{J}}\right)$ and $\left(Y^{i}\right)_{i \in \mathcal{J}}=\Phi\left(\left(Y^{i}\right)_{i \in \mathcal{J}}\right)$, we get the following comparison result for the two drivers of the RBSDE satisfied by $Y^{i}$ and $Y^{i, 1}$

$$
F_{i}\left(s, X_{s}^{t, x}, Y_{s}^{1}, \cdots, Y_{s}^{m}, z^{i}\right) \geq F_{i}\left(s, X_{s}^{t, x}, Y_{s}^{1,0}, \cdots, Y_{s}^{m, 0}, z^{i}\right)
$$

since $F_{i}$ is non-increasing w.r.t. all variables $y_{j}, j \in \mathcal{J}$. Next using the comparison result (see Remark 11) and uniqueness of the solution of the system associated with $\left(\left(F_{i}\left(s, X_{s}^{t, x}, y^{1}, \ldots, y^{m}, z\right)\right)_{i \in \mathcal{J}},\left(e^{\lambda T} h_{i}\left(X_{T}^{t, x}\right)\right)_{i \in \mathcal{J}},\left(e^{\lambda t} g_{i j}\left(s, X_{s}^{t, x}\right)\right)_{i, j \in \mathcal{J}}\right)$ we deduce that $Y^{i, 1} \leq Y^{i}$ for any $i \in \mathcal{J}$. Thus the property (5.22) is valid for $n=0$. Now if it is satisfied for some $n$ and repeating the same argumentation, it also holds for $n+1$, whence the claim. 
Next and relying once more on the result obtained El-Karoui et al.([12], Thm. 8.5) let $\tilde{v}^{i, n}, i \in \mathcal{J}$ and $n \geq 0$, be the deterministic continuous functions of $\Pi^{g}$ such that:

$$
\forall(t, x) \in[0, T] \times \mathbb{R}^{k}, \forall s \in[t, T], Y_{s}^{i, n}=\tilde{v}^{i, n}\left(s, X_{s}^{t, x}\right)
$$

First and as previously for any $i \in \mathcal{J}$, the sequence $\left(\tilde{v}^{i, n}\right)_{n \geq 0}$ is of Cauchy type and converges pointwisely to $v^{i}$. Next the inequalities (5.22) imply that: $\forall n \geq 0$ and $i \in \mathcal{J}$,

$$
\tilde{v}^{i, 2 n+1} \leq v^{i} \leq \tilde{v}^{i, 2 n}
$$

which implies

$$
v^{i}=\lim _{n} \nearrow \tilde{v}^{i, 2 n+1}=\lim _{n} \searrow \tilde{v}^{i, 2 n} .
$$

Therefore for any $i \in \mathcal{J}, v^{i}$ is both $u s c$ and $l s c$ and thus it is continuous. Next, since $\left(Y^{i}, Z^{i}, K^{i}\right)_{i \in \mathcal{J}}$ is the unique solution of the system of reflected BSDEs associated with the following triplet of datas $\left(\left(F_{i}\left(s, X_{s}^{t, x}, y^{1}, \ldots, y^{m}, z\right)\right)_{i \in \mathcal{J}},\left(e^{\lambda T} h_{i}\left(X_{T}^{t, x}\right)\right)_{i \in \mathcal{J}},\left(e^{\lambda t} g_{i j}\left(s, X_{s}^{t, x}\right)\right)_{i, j \in \mathcal{J}}\right)$ then $\left(\left(e^{-\lambda t} Y_{t}^{i}, e^{-\lambda t} Z_{t}^{i}, e^{-\lambda t} d K_{t}^{i}\right)_{t \leq T}\right)_{i \in \mathcal{J}}$ is the solution of the sytem of reflected BSDEs associated with $\left(\left(f_{i}\right)_{i \in \mathcal{J}},\left(h_{i}\right)_{i \in \mathcal{J}},\left(g_{i j}\right)_{i, j \in \mathcal{J}}\right)$ then using once more the result by El-Karoui et al. ([13], Thm.8.5) to deduce that $\left(e^{-\lambda t} v^{i}\right)_{i \in \mathcal{J}}$ is a continuous with polynomial growth solution of the system of variational inequalities with inter-connected obstacles (2.3). The proof is now complete.

Next we deal with the issue of uniqueness of the solution of (2.3) in the general case.

Theorem 8 Under Assumptions (H1),(H2),(H3) and (H4), the solution of the system of variational inequalities with inter-connected obstacles (2.3) is unique in the class $\left(v_{1}, \ldots ., v_{m}\right)$ of continuous functions which belong to $\Pi^{g}$.

Proof. As usual it is enough to show that if $\left(u_{1}, \ldots, u_{m}\right)\left(\operatorname{resp} .\left(v_{1}, \ldots, v_{m}\right)\right)$ is a continuous subsolution (resp. supersolution) of (2.3) such that $u_{i}, v^{i}, i \in \mathcal{J}$, belong to $\Pi^{g}$ then $u_{i} \leq v^{i}$, for all $i \in \mathcal{J}$. Thus classically we have uniqueness of (2.3) .

Step 1: We first assume the existence of a constant $\lambda<-m \cdot \max \left\{C_{f}^{j}, j=1, \ldots, m\right\}\left(C_{f}^{j}\right.$ is the Lipschitz constant of $f_{j}$ involved in [H2]-(ii)) such that for any $i \in \mathcal{J}, f_{i}$ verifies:

$\forall t, x, y_{1}, \ldots, y_{i-1}, y_{i+1}, \ldots, y_{m}, y, \bar{y}$, if $y \geq \bar{y}$ then

$$
f_{i}\left(t, x, y_{1}, \ldots, y_{i-1}, y, y_{i+1}, \ldots, y_{m}\right)-f_{i}\left(t, x, y_{1}, \ldots, y_{i-1}, \bar{y}, y_{i+1}, \ldots, y_{m}\right) \leq \lambda(y-\bar{y})
$$

Let $\gamma>0$ and $C$ be such that for any $i \in \mathcal{J}$ we have:

$$
\left|u_{i}(t, x)\right|+\left|v^{i}(t, x)\right| \leq C\left(1+|x|^{\gamma}\right), \forall(t, x) \in[0, T] \times \mathbb{R}^{k}
$$

Next as in Lemma 4.1, for $\theta>0$ and $\nu$ large enough $\left(v^{i}(t, x)+\theta e^{-\nu t}|x|^{2 \gamma+2}\right)_{i=1, m}$ is also a supersolution for (3.3). Therefore it is enough to show that for any $i \in \mathcal{J}$, we have:

$$
\forall(t, x) \in[0, T] \times \mathbb{R}^{k}, u_{i}(t, x) \leq v^{i}(t, x)+\theta e^{-\nu t}|x|^{2 \gamma+2},
$$


and taking the limit as $\theta \rightarrow 0$ we obtain the desired result. So let us set $w^{i, \theta, \nu}(t, x)=v^{i}(t, x)+$ $\theta e^{-\nu t}|x|^{2 \gamma+2},(t, x) \in[0, T] \times \mathbb{R}^{k}$ which is denoted by $w^{i}$ for simplicity. Assume now that there exists a point $(\bar{t}, \bar{x}) \in[0, T] \times \mathbb{R}^{k}$ such that $\max _{i \in \mathcal{J}}\left(u^{i}(\bar{t}, \bar{x})-w^{i}(\bar{t}, \bar{x})\right)>0$. Using the growth condition on $u_{i}$ and $w^{i}$, there exists $R>0$ such that:

$$
\forall i \in \mathcal{J}, \forall(t, x) \in[0, T] \times \mathbb{R}^{k} \text { s.t. }|x| \geq R, u_{i}(t, x)-w^{i}(t, x)<0 .
$$

Taking into account the values of the subsolution and the supersolution at $T$, it implies that

$$
\begin{aligned}
& 0<\max _{(t, x) \in[0, T] \times \mathbb{R}^{k}} \max _{i \in \mathcal{J}}\left(u_{i}(t, x)-w^{i}(t, x)\right)= \\
& \quad \max _{(t, x) \in[0, T[\times B(0, R)} \max _{i \in \mathcal{J}}\left(u_{i}(t, x)-w^{i}(t, x)\right)=\max _{i \in \mathcal{J}}\left(u_{i}\left(t^{*}, x^{*}\right)-w^{i}\left(t^{*}, x^{*}\right)\right)
\end{aligned}
$$

where $\left(t^{*}, x^{*}\right) \in[0, T[\times B(0, R)$. Now let $\tilde{\mathcal{J}}$ be as in $(4.5)$ and let $j \in \tilde{\mathcal{J}}$ be such that

$$
u_{j}\left(t^{*}, x^{*}\right)>\max _{k \in \mathcal{J}^{-j}}\left(u_{k}\left(t^{*}, x^{*}\right)-g_{j k}\left(t^{*}, x^{*}\right)\right) .
$$

Next let $\Phi_{n}^{j}(t, x, y)$ and $\varphi_{n}, n \geq 0$, be the same functions as in (4.6) and let $\left(t_{n}, x_{n}, y_{n}\right) \in[0, T] \times B^{\prime}(0, R)^{2}$ be the triple such that:

$$
\Phi_{n}^{j}\left(t_{n}, x_{n}, y_{n}\right)=\max _{(t, x, y) \in[0, T] \times B^{\prime}(0, R)^{2}} \Phi_{n}^{j}(t, x, y) .
$$

As in the proof of Theorem 4.1, one can show that:

$$
\left(t_{n}, x_{n}, y_{n}\right) \rightarrow\left(t^{*}, x^{*}, x^{*}\right) \text { and } n\left|x_{n}-y_{n}\right|^{2 \gamma+2} \rightarrow 0 \text { as } n \rightarrow \infty
$$

Now as $u_{j}$ and $g_{j k}$ are continuous functions and taking into account (5.25) we deduce that for $n$ large enough:

$$
u_{j}\left(t_{n}, x_{n}\right)>\max _{k \in \mathcal{J}^{-j}}\left(u_{k}\left(t_{n}, x_{n}\right)-g_{j k}\left(t_{n}, x_{n}\right)\right) .
$$

We next apply Crandall-Ishii-Lions's Lemma (see e.g. [8] or [14], pp.216) with $\Phi_{n}^{j}, u_{j}, w^{j}$ and $\varphi_{n}$ (recall that (5.27) is satisfied) at $\left(t_{n}, x_{n}, y_{n}\right)$, there exist $\left(p_{u}^{n}, q_{u}^{n}, M_{u}^{n}\right) \in \bar{J}^{2,+}\left(u_{j}\right)\left(t_{n}, x_{n}\right)$ and $\left(p_{w}^{n}, q_{w}^{n}, M_{w}^{n}\right) \in$ $\bar{J}^{2,-}\left(w^{j}\right)\left(t_{n}, y_{n}\right)$ such that:

$$
\begin{gathered}
p_{u}^{n}-p_{w}^{n}=\partial_{t} \tilde{\varphi}_{n}\left(t_{n}, x_{n}, y_{n}\right)=2\left(t_{n}-t^{*}\right), q_{u}^{n}\left(\text { resp. } q_{w}^{n}\right)=\partial_{x} \varphi_{n}\left(t_{n}, x_{n}, y_{n}\right)\left(\text { resp. }-\partial_{y} \varphi_{n}\left(t_{n}, x_{n}, y_{n}\right)\right) \text { and } \\
\qquad\left(\begin{array}{ll}
M_{u}^{n} & 0 \\
0 & -N_{w}^{n}
\end{array}\right) \leq A_{n}+\frac{1}{2 n} A_{n}^{2}
\end{gathered}
$$

where $A_{n}=D_{(x, y)}^{2} \varphi_{n}\left(t_{n}, x_{n}, y_{n}\right)$. Taking now into account that $\left(u_{i}\right)_{i \in \mathcal{J}}\left(\right.$ resp. $\left.\left(w^{i}\right)_{i \in \mathcal{J}}\right)$ is a subsolution (resp. supersolution) of (2.3) and once more (5.27) we deduce:

$$
-p_{u}^{n}-b\left(t_{n}, x_{n}\right)^{\top} \cdot q_{u}^{n}-\frac{1}{2} \operatorname{Tr}\left[\left(\sigma \sigma^{\top}\right)\left(t_{n}, x_{n}\right) M_{u}^{n}\right]-f_{j}\left(t_{n}, x_{n},\left(u_{i}\left(t_{n}, x_{n}\right)\right)_{i \in \mathcal{J}}, \sigma\left(t_{n}, x_{n}\right)^{\top} \cdot q_{u}^{n}\right) \leq 0
$$

and

$$
-p_{w}^{n}-b\left(t_{n}, y_{n}\right)^{\top} \cdot q_{w}^{n}-\frac{1}{2} \operatorname{Tr}\left[\left(\sigma \sigma^{\top}\right)\left(t_{n}, y_{n}\right) M_{w}^{n}\right]-f_{j}\left(t_{n}, y_{n},\left(w^{i}\left(t_{n}, y_{n}\right)\right)_{i \in \mathcal{J}}, \sigma\left(t_{n}, y_{n}\right)^{\top} \cdot q_{w}^{n}\right) \geq 0 .
$$


Making the difference between those two inequalities yields:

$$
\begin{aligned}
& -\left(p_{u}^{n}-p_{w}^{n}\right)-\left(b\left(t_{n}, x_{n}\right)^{\top} \cdot q_{u}^{n}-b\left(t_{n}, y_{n}\right)^{\top} \cdot q_{w}^{n}\right)-\frac{1}{2} \operatorname{Tr}\left[\left\{\sigma \sigma^{\top}\left(t_{n}, x_{n}\right) M_{u}^{n}-\sigma \sigma^{\top}\left(t_{n}, y_{n}\right) M_{w}^{n}\right\}\right] \\
& \quad-\left\{f_{j}\left(t_{n}, x_{n},\left(u_{i}\left(t_{n}, x_{n}\right)\right)_{i \in \mathcal{J}}, \sigma\left(t_{n}, x_{n}\right)^{\top} \cdot q_{u}^{n}\right)-f_{j}\left(t_{n}, y_{n},\left(w^{i}\left(t_{n}, y_{n}\right)\right)_{i \in \mathcal{J}}, \sigma\left(t_{n}, y_{n}\right)^{\top} \cdot q_{w}^{n}\right)\right\} \leq 0
\end{aligned}
$$

and then

$$
-\left\{f_{j}\left(t_{n}, x_{n},\left(u_{i}\left(t_{n}, x_{n}\right)\right)_{i \in \mathcal{J}}, \sigma\left(t_{n}, x_{n}\right)^{\top} \cdot q_{u}^{n}\right)-f_{j}\left(t_{n}, x_{n},\left(w^{i}\left(t_{n}, y_{n}\right)\right)_{i \in \mathcal{J}}, \sigma\left(t_{n}, x_{n}\right)^{\top} \cdot q_{u}^{n}\right)\right) \leq A_{n}
$$

where as in (4.12) and (4.13) we have $\varlimsup_{n \rightarrow \infty} A_{n} \leq 0$. Next linearizing $f_{j}$, which is Lipschitz w.r.t. $\left(y^{i}\right)_{i \in \mathcal{J}}$, and using (5.23) we obtain:

$$
-\lambda\left(u_{j}\left(t_{n}, x_{n}\right)-w^{j}\left(t_{n}, y_{n}\right)\right)-\sum_{k \in \mathcal{J}^{-j}} \Theta_{n}^{j, k}\left(\left(u_{k}\left(t_{n}, x_{n}\right)-w^{k}\left(t_{n}, y_{n}\right)\right) \leq A_{n}\right.
$$

with $\Theta_{n}^{j, k}$ which stands for the increment rate of $f_{j}$ w.r.t. $y_{k}$ and which, thanks to the monotonicity assumption (see [H2] (iv)), is nonnegative and bounded by $C_{f}^{j}$ the Lipschitz constant of $f_{j}$. Thus

$$
\begin{aligned}
-\lambda\left(u_{j}\left(t_{n}, x_{n}\right)-w^{j}\left(t_{n}, y_{n}\right)\right) & \leq \sum_{k \in \mathcal{J}^{-j}} \Theta_{n}^{j, k}\left(\left(u_{k}\left(t_{n}, x_{n}\right)-w^{k}\left(t_{n}, y_{n}\right)\right)^{+}+A_{n}\right. \\
& \leq C_{f}^{j} \sum_{k \in \mathcal{J}^{-j}}\left(\left(u_{k}\left(t_{n}, x_{n}\right)-w^{k}\left(t_{n}, y_{n}\right)\right)^{+}+A_{n} .\right.
\end{aligned}
$$

Taking now the limit as $n \rightarrow \infty$ and since $j \in \tilde{\mathcal{J}}$ to obtain:

$$
\begin{aligned}
-\lambda\left(u_{j}\left(t^{*}, x^{*}\right)-w^{j}\left(t^{*}, x^{*}\right)\right) & \leq C_{f}^{j} \sum_{k \in \mathcal{J}^{-j}}\left(u_{k}\left(t^{*}, x^{*}\right)-w^{k}\left(t^{*}, x^{*}\right)\right)^{+} \\
& \leq(m-1) C_{f}^{j}\left(u_{j}\left(t^{*}, x^{*}\right)-w^{j}\left(t^{*}, x^{*}\right)\right)
\end{aligned}
$$

which contradictory since $u_{j}\left(t^{*}, x^{*}\right)-w^{j}\left(t^{*}, x^{*}\right)>0$ and $-\lambda>m C_{f}^{j}$. Thus for any $i \in \mathcal{J}, u_{i} \leq w^{i}$.

Step 2: The general case.

For arbitrary $\lambda \in \mathbb{R}$, if $\left(u_{j}\right)_{j \in \mathcal{J}}$ (resp. $\left(v^{j}\right)_{j \in \mathcal{J}}$ ) is a subsolution (resp. supersolution) of (2.3) then $\tilde{u}_{j}(t, x)=e^{\lambda t} u_{j}(t, x)$ and $\tilde{v}^{j}(t, x)=e^{\lambda t} v^{j}(t, x)$ is a subsolution (resp. supersolution) of the following system of variational inequalities with oblique reflection (see the proof of Proposition 4.1, Step 2): $\forall i \in \mathcal{J}$,

$$
\left\{\begin{array}{l}
\min \left\{\tilde{v}_{i}(t, x)-\max _{j \in \mathcal{J}^{-i}}\left(-e^{\lambda t} g_{i j}(t, x)+\tilde{v}_{j}(t, x)\right)\right. \\
\left.\quad-\partial_{t} \tilde{v}_{i}(t, x)-\mathcal{L} \tilde{v}_{i}(t, x)+\lambda \tilde{v}_{i}(t, x)-e^{\lambda t} f_{i}\left(t, x,\left(e^{-\lambda t} \tilde{v}^{i}(t, x)\right)_{i \in \mathcal{J}}, e^{-\lambda t} \sigma^{\top}(t, x) \cdot D_{x} \tilde{v}^{i}(t, x)\right)\right\}=0 ; \\
\tilde{v}_{i}(T, x)=e^{\lambda T} h_{i}(x) .
\end{array}\right.
$$

But in choosing $\lambda$ small enough the functions $F_{i}\left(t, x,\left(u_{i}\right)_{i \in \mathcal{J}}, z\right)=-\lambda u+e^{\lambda t} f_{i}\left(t, x,\left(e^{-\lambda t} u_{i}\right)_{i \in \mathcal{J}}, e^{-\lambda t} z\right)$, $i \in \mathcal{J}$, satisfy condition (5.23) and then thanks to the result stated in Step 1, we have $\tilde{u}_{i} \leq \tilde{v}_{i}, i \in \mathcal{J}$. Thus $u_{i} \leq v_{i}$ for any $i \in \mathcal{J}$ which is the desired result.

As a by-product we have:

Corollary 2 Assume that (H1), (H3), (H4) and (H2) are fulfilled, then there is a unique solution of the system (2.3) in the class of continuous functions with polynomial growth. 


\section{References}

[1] T. Arnarsson, B. Djehiche, M. Poghosyan, H. Shahgholian: A PDE approach to regularity of solutions to finite horizon optimal switching problmes. Nonlinear Analysis, Series A: Theory, Methods and Applications, 2009.

[2] Bernhart, M.: Modélisation et méthodes d'évaluation de contrats gaziers: Approches par contrôle stochastique, PHD Thesis, Université Denis Diderot, P.7, 2011.

[3] Bouchard, B.: A stochastic target formulation for optimal switching problems in finite horizon, Stochastics, 81 (2): 171-197, 2009.

[4] Brennan,M. and Schwarz, E.S.: Evaluating natural resource investment, J. Business, 58: 135-137, 1985.

[5] Carmona, R., Ludkovski, M.: Pricing asset scheduling flexibility using optimal switching, Appl. Math. Finance, 15 (4): 405-447, 2008.

[6] Carmona, R., Ludkovski, M.: Valuation of energy storage: an optimal switching approach, Quantitative finance, 10 (4): 359-374, 2010.

[7] Chassagneux, J.-F., Elie R. and Kharroubi I.: A note on existence and uniqueness for solutions of multidimensional reflected BSDEs, Electron. Commun. Probab. 16, pp. 120-128, 2011.

[8] Crandall, M.G. Ishii, H. and Lions, P.-L.: User's guide for viscosity solutions, Bulletin of the American Mathematical Society, 27(1): 1-67, 1992.

[9] Dixit, A.: Entry and exit decisions under uncertainty, J. Political economy, 97: 620-623, 1989

[10] Djehiche, B., Hamadène S. and Popier, A.: A finite horizon optimal multiple switching problem, SIAM Journal Control and Optim, 48(4): 2751-2770, 2009.

[11] Elie, R. and Kharroubi, I.: Probabilistic Representation and Approximation for couples systems of variational inequalities, Statistics and Probability Letters, 80: 1388-1396, 2010.

[12] El Karoui,N., Kapoudjan, C., Pardoux, E., Peng,.S. and Quenez, M.-C.: Reflected solutions of backward SDE's and related problems for PDE's, Ann. Prob., 25(2): 702-737, 1997.

[13] El Karoui,N., Peng,.S. and Quenez, M.-C.: Backward SDEs in Finance, Mathematical Finance, 7 (1): 1-71, 1997.

[14] Fleming, W. H., and Soner, M.: Controlled Markov processes and viscosity solutions, Applications of Mathematics, Stochasting modelling and applied probability, 25, Springer-Verlag, 2006.

[15] El Asri, B. and Hamadène S.: The finite horizon optimal multi-modes switching problem: the viscosity solution approach, Appl. Math. Optim, 60: 213-235, 2009.

[16] Hamadène, S.: Reflected BSDE's with Discontinuous Barriers and application Stochastics and Stochastics Reports, 74 (3-4): 571-596, 2002.

[17] Hamadène, S., Hassani, M.: BSDEs with two reflecting barriers: the general result, Probab Theory Relat. Fields, 132: 237-264 2005. 
[18] Hamadène, S and Jeanblanc, M.: On the Starting and Stopping Problem: Application in reversible investments, Math of Operation Research, 32 (1) : 182-192, 2007.

[19] Hamadène, S., and Zhang, J.: Switching problem and related system of reflected backward stochastic differential equations Stoch. Proc. and their applications, 120: pp.403-426,2010.

[20] Hu Y., and Tang, S.: Multi-dimensional BSDE with oblique reflection and optimal switching, Proba. Theo. and Rel. Fields, 147, N. 1-2: 89-121, 2010.

[21] Ishii, H. and Koike, K.: Viscosity solutions of a system of Nonlinear second order PDE's arising in switching games, Functional, 34, 143-155, 1991.

[22] Ludkowski, M.: Stochastic Switching Games and Duopolistic Competition in Emissions Markets, SIAM Journal on Financial Mathematics, 2, pp. 488-511, 2011.

[23] Kharroubi, I., Ma, J., Pham H. and Zhang J.: Backward SDEs with constrained jumps and quasi variational inequalities, The Annals of Probab., 38(2): 794-840, 2010.

[24] Pardoux, E. and Peng,S.: Adapted solution of a backward stochastic differential equation, Systems and Control Letters 14: 55-61, 1990.

[25] Peng, S.: Monotonic limit theorem of BSDE and nonlinear decomposition theorem of Doob Meyers type, Probab theory and Rel. fields, 113(4), 473-499, 1999.

[26] Pham, H.: Continuous-time stochastic control and optimization with financial applications, Stochastic modelling and applied prob., Springer-verlag, Berlin, 2009.

[27] Porchet, A., Touzi, N.,Warin, X.: Valuation of a power plant under production constraints and market incompleteness, Math. Methods Oper. Res., 70(1): 45-75, 2009.

[28] Tang, S., Yong, J.: Finite horizon stochastic optimal switching and impulse controls with a viscosity solution approach, Stoch. Stoch. Rep. 45: 145-176, 1999.

[29] Zervos, M. : A Problem of Sequential Entry and Exit Decisions Combined with Discretionary Stopping, SIAM J. Control Optim., 42(2), 397-421, 2003. 